OPEN ACCESS

Edited by:

Nicola Maggio

Sheba Medical Center, Israe

Reviewed by:

Pavel M. Balaban,

Institute of Higher Nervous Activity

and Neurophysiology (RAS), Russia

Michael Fox,

Virginia Tech Carilion Research Institute, United States

${ }^{*}$ Correspondence:

Lars Kunz

Lars.Kunz@bio.Imu.de

Received: 21 November 2017 Accepted: 05 April 2018 Published: 24 April 2018

Citation:

Keplinger $S$, Beiderbeck $B$ Michalakis S, Biel M, Grothe B and Kunz L (2018) Optogenetic Control of Neural Circuits in the Mongolian Gerbil. Front. Cell. Neurosci. 12:111. doi: 10.3389/fncel.2018.00111

\section{Optogenetic Control of Neural Circuits in the Mongolian Gerbil}

\author{
Stefan Keplinger ${ }^{1}$, Barbara Beiderbeck ${ }^{1,2}$, Stylianos Michalakis ${ }^{3}$, Martin Biel $^{3}$, \\ Benedikt Grothe ${ }^{1}$ and Lars Kunz ${ }^{1 *}$
}

\begin{abstract}
${ }^{1}$ Division of Neurobiology, Department Biology II, Biocenter, Ludwig Maximilian University of Munich, Munich, Germany, ${ }^{2}$ Graduate School of Systemic Neurosciences, GSN-LMU, Ludwig Maximilian University of Munich, Munich, Germany, ${ }^{3}$ Center for Integrated Protein Science Munich (CiPSM), Department of Pharmacy, Center for Drug Research, Ludwig Maximilian University of Munich, Munich, Germany
\end{abstract}

The Mongolian gerbil (Meriones unguiculatus) is widely used as a model organism for the human auditory system. Its hearing range is very similar to ours and it uses the same mechanisms for sound localization. The auditory circuits underlying these functions have been characterized. However, important mechanistic details are still under debate. To elucidate these issues, precise and reversible optogenetic manipulation of neuronal activity in this complex circuitry is required. However, genetic and genomic resources for the Mongolian gerbil are poorly developed. Here, we demonstrate a reliable gene delivery system using an AAV8(Y337F)-pseudotyped recombinant adeno-associated virus (AAV) 2-based vector in which the pan-neural human synapsin (hSyn) promoter drives neuron-specific expression of $\mathrm{CatCH}\left(\mathrm{Ca}^{2+}\right.$-permeable channelrhodopsin) or NpHR3.0 (Natronomonas pharaonis halorhodopsin). After stereotactic injection into the gerbil's auditory brainstem (medial nucleus of the trapezoid body, dorsal nucleus of the lateral lemniscus) and midbrain [inferior colliculus (IC)], we characterized $\mathrm{CatCH}$ - and/or NpHR3.0-transduced neurons in acute brain slices by means of whole-cell patchclamp recordings. As the response properties of optogenetic tools strongly depend on neuronal biophysics, this parameterization is crucial for their in vivo application. In a proof-of-principle experiment in anesthetized gerbils, we observed strong suppression of sound-evoked neural responses in the dorsal nucleus of the lateral lemniscus (DNLL) and IC upon light activation of NpHR3.0. The successful validation of gene delivery and optogenetic tools in the Mongolian gerbil paves the way for future studies of the auditory circuits in this model system.

Keywords: auditory, brainstem, AAV, MNTB, DNLL, IC, CatCH, NpHR3.0

\section{INTRODUCTION}

The discovery of channelrhodopsins and light-activated $\mathrm{Cl}^{-}$pumps and their application as highly versatile optogenetic tools for controlling light-stimulated excitation and inhibition of neurons have made many exciting neurobiological discoveries possible over the past decade (Boyden et al., 2005; Nagel et al., 2005; Deisseroth et al., 2006; Zhang et al., 2010; Fenno et al., 2011;

Abbreviations: $\mathrm{AAV}$, adeno-associated virus; $\mathrm{AP}$, action potential; $\mathrm{CatCH}, \mathrm{Ca}^{2+}$-permeable channelrhodopsin; $\mathrm{ChR}$, channelrhodopsin 2; D-AP5, D-(-)-2-amino-5-phosphonopentanoic acid; DNLL, dorsal nucleus of the lateral lemniscus; DNQX, 6,7-dinitroquinoxaline-2,3-dione; hSyn, human synapsin; IC, inferior colliculus; LSO, lateral superior olive; MAP2, microtubule-associated protein 2; MNTB, medial nucleus of the trapezoid body; MSO, medial superior olive; NpHR3.0, Natronomonas pharaonis halorhodopsin 3.0; P, post-natal day; PBS, phosphate-buffered saline; SNR, signal to noise ratio; WPRE, woodchuck hepatitis virus posttranscriptional regulatory element. 
Yizhar et al., 2011a). Since the application of optogenetics requires gene transfer, most in vivo studies of neural networks have been performed in the mouse, for which methods of gene transfer and genome manipulation are well established. For model organisms such as the Mongolian gerbil (Meriones unguiculatus), the use of optogenetic tools is far less well developed. The Mongolian gerbil is the model of choice for human hearing research because - unlike the case in mice and rats - its audiogram includes most of the human low-frequency hearing range (Ryan, 1976) and age-related hearing loss occurs in both species (Mills et al., 1990). The circuitry underlying sound source localization has been well described in vitro and in vivo and resembles that found in humans (Spitzer and Semple, 1991, 1995; Tollin, 2003; Maki and Furukawa, 2005; Siveke et al., 2006; Pecka et al., 2008; Kandler et al., 2009; Lingner et al., 2012; Grothe and Pecka, 2014; Roberts et al., 2014; Manley, 2017). Unfortunately, annotation of the gerbil genome is only now on its way (Zorio et al., 2018). and techniques for the generation of transgenic or knock-out strains are difficult because breeding is time consuming and reproductive performance is poor compared to mice (Ågren, 1984; Salo and French, 1989).

The in vivo application of optogenetic tools to the Mongolian gerbil would enable us to understand functions of auditory circuits by reversibly activating or silencing defined brain nuclei (Willaredt et al., 2015; Nothwang, 2016). In the context of sound source localization, the ability to optogenetically address the MNTB, the dorsal nucleus of lateral lemniscus (DNLL) and the IC would be a huge step forward, as these nuclei are crucial for auditory functions. The MNTB is central to sound source localization because it provides glycinergic inhibition to the MSO and the LSO. Moreover, it represents a good example for a nucleus in which direct electrical stimulation of neurons is impossible, because large numbers of fibers projecting to the MSO and LSO pass through it and would be electrically activated as well. The DNLL has been hypothesized to be crucial in the context of echo suppression and the precedence effect (Cremer, 1948; Wallach et al., 1949; Haas, 1951; Shneiderman et al., 1988; Ito et al., 1996; Kelly et al., 1996; Pecka et al., 2007; Ammer et al., 2015). The IC is a central structure in sound processing (Pollak et al., 2003). It represents an ideal model nucleus because it is easily accessible to both virus injection and light application, due to its dorsal location.

In order to express channelrhodopsins and halorhodopsins in designated auditory nuclei, we chose recombinant AAV vectors as a reliable gene delivery system. AAV vectors are easy to produce in high titers and safe to use owing to their lack of pathogenicity, low immunogenicity, replication deficiency and low biological safety level. More than 12 naturally occurring subtypes have been described, which display distinct tropisms for certain cell types (Burger et al., 2004; Zincarelli et al., 2008; Aschauer et al., 2013). Among the various serotypes, AAV8 and AAV9 transduce neurons at very high rates (Broekman et al., 2006; Masamizu et al., 2011). Moreover, phosphorylation of surface-exposed tyrosine residues in the AAV capsid has been reported to contribute to targeting to the proteasome for degradation (Zhong et al., 2008). Accordingly, AAV vectors with mutated tyrosine residues may enhance levels of gene expression. To restrict gene expression to neuronal cells, we used the 485-bp hSyn promoter. AAVs have previously been used to transduce regions in the frontal cortex and the hippocampus in the gerbil (Shimazaki et al., 2000; Bellomo et al., 2006). In a recent study, an AAV9 vector bearing an NpHR gene (see below) was utilized to silence firing of the trigeminal nerve in the gerbil brainstem (Chen et al., 2017). However, nuclei in the midbrain or brainstem of gerbils have remained inaccessible to optogenetic manipulations. We chose AAV8(Y337F), an AAV8 serotype with a single tyrosine-to-phenylalanine mutation at position 733 in the AAV viral protein 1 (VP1), as the vehicle for gene transfer. The vector of choice must provide for: (1) specific and efficient transduction of neurons in the auditory midbrain and brainstem, (2) reliable expression of a functional transgene in neurons, (3) fast ( $\leq 2$ weeks), stable and high-level gene expression to permit manipulation of the membrane potential upon expression of optogenetic tools, and (4) transduction of sufficiently high numbers of neurons (i.e., at least 30-50\%) within the injected area.

For neuronal excitation, we utilized the L132C mutant ChR2 variant $\mathrm{CatCH}\left(\mathrm{Ca}^{2+}\right.$ translocating channelrhodopsin) (Kleinlogel et al., 2011). CatCH exhibits a three-fourfold increase in photocurrent relative to wild-type ChR2, due to a shift in the balance of the pore's ion selectivity filter in favor of $\mathrm{Ca}^{2+}$, a small shift in absorption maximum (474 nm), a $>10$-fold increase in light sensitivity, and fast kinetics $\left(\tau_{\text {off }}=16 \mathrm{~ms}\right)$. For inhibition, we used the light-driven chloride pump NpHR3.0, which is based on the $\mathrm{NpHR}$, the halorhodopsin isolated from the archeal halobacterium Natronomonas pharaonis (Han and Boyden, 2007; Zhang et al., 2007). It has an absorption maximum at $590 \mathrm{~nm}$ and hyperpolarizes the cell membrane by pumping chloride into the cytoplasm. Targeting of NpHR3.0 to the plasma membrane was improved and its aggregation prevented by adding the trafficking signal from $\mathrm{K}_{\mathrm{ir}} 2.1$, an inward rectifying $\mathrm{K}^{+}$channel (Gradinaru et al., 2010). NpHR3.0 features an increased photocurrent of $\sim 750 \mathrm{pA}$, fast kinetics with a $\tau_{\text {off }}$ of $\sim 4.2 \mathrm{~ms}$ and a 20 -fold increase in light sensitivity compared to the wild-type NpHR. Following successful gene delivery and expression, CatCH and NpHR3.0 needed to be characterized in vitro to determine the appropriate expression times, success rate, optimal light stimulation protocols, the kinetics of antagonist action and their effects on neuronal spiking. These issues were addressed by obtaining whole-cell patch-clamp recordings from transduced neurons in acute brain slices. In a final proof-of-principle experiment involving extracellular in vivo recordings, sound-evoked neuronal spiking in the IC and the DNLL of anesthetized animals was suppressed by light in neurons expressing NpHR3.0.

\section{MATERIALS AND METHODS}

\section{Gerbils}

Ethical approval was obtained for the animal work. The experiments were carried out in accordance with regional regulations, national law (German Animal Welfare Act) and the Council of the European Union's Directive 2010/63/EU. 
All experiments were approved by the District Government of Upper Bavaria ('Regierung von Oberbayern'; Ref. No. 55.2-1-542531-105-10). Mongolian gerbils (M. unguiculatus) were bred in the certified in-house breeding facility at LMU Munich (German Animal Welfare Act, 4.3.2-5682/LMU/Department Biology II). Gerbils of either sex were injected with AAVs on postnatal day (P) 28-60 for in vivo recordings and immunohistological analyses and on P 2-30 for in vitro experiments (Keplinger, 2016).

\section{Construction of hSyn-CatCH-mCherry-WPRE}

pAAV2-ss-hSyn-hChR(H134R)-mCherry-WPRE (obtained from Dr. Karl Deisseroth) served as backbone for vector construction and was cleaved with SalI and HindIII (New England Biolabs Inc.). The DNA coding for CatCH (from Dr. Sonja Kleinlogel) was PCR-amplified with the primers 5'-TATAGTCGACATGGATTATGGAGGCGC-3' and 5'-GGC TGGCGCGGTACCCAAGCTTAT- $3^{\prime}$. The PCR product was cleaved with SalI and HindIII, and ligated into the corresponding sites in the dephosphorylated vector plasmid. The ligation mixture was transformed into Escherichia coli 10-beta cells and positive transformants were selected by restriction fragment length analysis. The intact plasmid pAAV2-ss-hSyn-CatCH-WPRE was then cleaved with HindIII and dephosphorylated. mCherry was PCR-amplified with the primers 5'-TATAAAGCTTATGGTGAGCAAGGGCG-3' and 5'-CGAGCTGTACAAGTAAAAGCTTAT-3'. The PCR product was digested with HindIII and ligated into the same site in pAAV2-ss-hSyn-CatCH-[/]-WPRE. The ligation was transformed into the E. coli 10-beta, and positive clones with the correct plasmid were identified by digesting isolated DNA.

\section{Construction of pAAV2.1-ss-hSyn-Catch- mCherry-T2A-NpHR3.0-WPRE}

The donor plasmid described above was digested with SalI and EcoRI, and the new insert CatCH-mCherry-T2A-NpHR was constructed by fusion PCR. The three parts of the insert, CatCHmCherry, T2A and NpHR3.0 were amplified by PCR. CatCHmCherry was amplified with the primers $5^{\prime}$-TATAGTCGACAT GGATTATGGAGGCGC-3' ${ }^{\prime}$ and 5' -CTTGTACAGCTCGTCCAT GCC-3'. T2A DNA was amplified with the primers $5^{\prime}$ GCGGCATGGACGAGCTGTACAAGGCCACGAACTTCTCT CTGTTAAA- ${ }^{\prime}$ and 5'-AGGACCGGGGTTTTCTTCCA- ${ }^{\prime}$. NpHR3.0 was amplified with the primers $5^{\prime}$-GACGTGGAA GAAAACCCCGGTCCTATGACAGAGACCCTGCCTCCC-3' and $5^{\prime}$ - CTCTGAATTCTTTACACCTCGTTCTCGTAGCAGA ACACGTTGATGTCGATCTGGTCC-3'. All three amplicons were purified by agarose gel electrophoresis. T2A and NpHR3.0 were fused together using the primers $5^{\prime}$-GCGGCATGGACGA GCTGTACAAG GCCACGAACTTCTCTCTGTTAAA-3' and 5'-CTCTGAATTCTTTACACCTCGTTCTCGTAGCAGAACAC GTTGATGTCGATCTGGTCC-3'. CatCH-mCherry and T2ANpHR3.0 were fused with the primers $5^{\prime}$-TAGAGTCGACACTA TGGCGGCGCTTTGTCTG and 5'-CTCTGAATTCTTTACAC CTCGTTCTCGTAGCAGAACACGTTGATGTCGATCTGGTC C-3'. The resulting amplicon CatCH-mCherry-T2A-NpHR3.0 was then purified by agarose gel electrophoresis and subcloned into the cloning vector pJet1.2. The ligation was transformed into $E$. coli 10-beta. The insert was then cut out of its host vector with SalI and EcoRI, and ligated into the cleaved donor plasmid.

\section{AAV Vector Production}

The cis plasmids pAAV2.1-ss-hSyn-EYFP-WPRE, pAAV2.1ss-hSyn-CatCH-mCherry-WPRE, pAAV2.1-ss-hSyn-NpHR3.0EYFP-WPRE, pAAV2.1-ss-hSyn-Catch-mCherry-T2A-NpHR3.0 -WPRE, and pAAV2.1-sc-hSyn-NpHR3.0-mCherry-SV40 were used to produce single-stranded AAV8(Y733F)-pseudotyped AAV2 vectors according to published procedures (Koch et al., 2012; Becirovic et al., 2016). Briefly, 293T cells were transfected with the trans plasmids pAdDeltaF6, and pAAV2/8Y733F, and the appropriate cis plasmid, using the calcium phosphate method. rAAV2/8Y7333F particles were harvested after $48 \mathrm{~h}$ and purified on iodixanol gradients. The $40-60 \%$ iodixanol interface was further purified and concentrated by ion-exchange chromatography on a 5-ml HiTrap Q Sepharose column using the ÄKTA Basic FPLC system (GE Healthcare, Munich, Germany) according to previously described procedures, followed by further concentration using Amicon Ultra-4 Centrifugal Filter Units (Millipore, Schwalbach, Germany). Physical titers (in genome copies/ml) were determined by quantitative PCR on a LightCycler 480 (Roche Applied Science, Mannheim, Germany) using the KAPPA SYBR FAST kit (Peqlab, Erlangen, Germany) and the following primer set: WPREF: 5'-AGTTGTGGCCCGTTGTCAGG$3^{\prime}$ and WPRER: 5'-AGTTCCGCCGTGGCAATAGG-3'. In all our experiments, the vector with the DNA backbone of the AAV2 serotype was packaged in the capsid of the rAAV2/8Y733F, and in the following this combination is referred to as $\mathrm{AAV} 8(\mathrm{Y} 733 \mathrm{~F})$.

\section{AAV Injection}

Gerbils were anesthetized by subcutaneous injection with medetomidine (Domitor, Pfizer Inc.), midazolam (Midazolam, Ratiopharm GmbH) and fentanyl (Fentanyl, Janssen Pharmaceutica), and additionally injected with meloxicam (Metacam) and Ringer's solution (B. Braun Melsungen AG). Anesthetized animals were placed on a custom-made heating pad and the depth of anesthesia was monitored by testing the paw pinch reflex. An incision was made from lambda to $1 \mathrm{~cm}$ rostral of the bregma and a custom-made head-fixating device was glued onto the skull directly rostral to the bregma. Gerbils were then head fixed in a custom-made stereotaxic frame (Schuller et al., 1986), which allowed motorized movement in $\mathrm{X}$-, Y- and Z-direction by micro manipulators (Junior XYZ-R, Luigs \& Neumann Feinmechanik und Elektrotechnik GmbH) controlled by a XYZ axis controller (SM-5, Luigs \& Neumann Feinmechanik und Elektrotechnik $\mathrm{GmbH}$ ). The skull was aligned relative to lambda and bregma. A small 2-mm hole was drilled into the skull without damaging the dura mater. Injection needles with an inner diameter of $30-40 \mu \mathrm{m}$ at the tip were pulled with a vertical glass electrode puller (PE-2, Narishige Ltd.) from glass capillaries (ID $=0.530 \mathrm{~mm} \pm 25 \mu \mathrm{m}$, OD $1.14 \mathrm{~mm}$, Item\#: 4878, World Precision Instruments Inc.). 
They were filled with mineral oil, inserted into the micro injector (Nanoliter 2000, World Precision Instruments Inc.) and front-filled with $1 \mu \mathrm{l} \mathrm{rAAV}$ (diluted to $9.5-9.8 \times 10^{8} \mathrm{gc} / \mu \mathrm{l}$ ). The position of the tip was then zeroed at lambda and lowered with $10 \mu \mathrm{m} / \mathrm{sec}$ to the respective coordinates (relative to lambda: IC $x=1900 \mu \mathrm{m}, y=0 \mu \mathrm{m}, z=-3900 /-4000 \mu \mathrm{m}$, injector angle: $20^{\circ}$ to rostral; DNLL $x=1750 \mu \mathrm{m}, y=-900 \mu \mathrm{m}$, $z=-5400 /-5500 \mu \mathrm{m}$, injector angle: $4-5^{\circ}$ to medial; MNTB $x=1050 \mu \mathrm{m}, y=950 \mu \mathrm{m}, z=-8050 /-8150 \mu \mathrm{m}$, injector angle $12^{\circ}$ to rostral). The injection (final volume $250 \mathrm{nl}$ per injection spot) was delivered at a rate of $0.92 \mathrm{nl} / \mathrm{sec}$. After $5 \mathrm{~min}$, the needle was retracted $(10 \mu \mathrm{m} / \mathrm{sec})$, the gerbil was removed from the stereotaxic frame and the incision was sutured (Suprama HS18 USP 4/0, Feuerstein GmbH) and glued with histoacryl. Anesthesia was antagonized with atipamezole (Antisedan, Orion Pharma), flumazenil (Anexate, Roche Pharma AG) and naloxone (Naloxon, Hameln Pharma Plus GmbH), and Ringer's solution was administered by subcutaneous injection. The gerbils were monitored closely until recovery and meloxicam was administered at 24-h intervals for at least 3 days after surgery. The success rates for injection into the target nuclei, i.e., the presence of transduced neurons afterwards, were $100 \%$ for the IC $(n=12 / 12), 33 \%$ for the DNLL $(n=1 / 3)$ and $33 \%$ for the MNTB $(n=1 / 3)$.

\section{Immunohistochemistry and Confocal Microscopy}

Immunohistochemistry was carried out on tissue obtained from animals between $\mathrm{P}$ 28-60. The animals were injected with a lethal dose of pentobarbital [Narcoren (160 g/l), Merial GmbH; $2 \mathrm{mg} / \mathrm{kg}$ body mass] and perfused with PBS containing $0.1 \%$ heparin and $155 \mathrm{mM} \mathrm{NaCl}$, followed by perfusion with $4 \%$ paraformaldehyde (PFA). After perfusion, the brain was removed from the skull and post-fixed in 4\% PFA either overnight for vibratome sectioning or for $2-4 \mathrm{~h}$ for cryosectioning. In the case of cryosectioning, the brains were incubated in $20 \%$ sucrose solution (diluted in $0.02 \mathrm{M}$ PBS) overnight to prevent solidification after freezing. They were then washed in $0.02 \mathrm{M}$ $\mathrm{PBS}$ and deep-frozen using high-pressure $\mathrm{CO}_{2}$. Cryosections of 50- $\mu \mathrm{m}$ thickness were cut using a Leica CM3050 S cryostat. For vibratome sectioning brains were washed twice in $0.1 \mathrm{M}$ PBS and slices of $50-\mu \mathrm{m}$ thickness were cut with a VT1200S (Leica).

Standard immunohistochemistry procedures were used to stain free-floating slices with primary antibodies for $\mathrm{NeuN}$ (Millipore, MAB377), S100B (SWANT, 37A), and MAP2 (Neuromics, CH22103). Secondary antibodies were applied the following day for $2 \mathrm{~h}$ at room temperature. These were conjugated with Alexa 488 (Molecular Probes, Invitrogen), Cy3 (Dianova) or Cy5 (Dianova). Slices were mounted in Vectashield medium (H-1200, Vector Laboratories) and confocal scans were taken with a Leica SP5 System. Images were acquired with 8-bit intensity resolution using a HCX PL APO CS 20.0x0.70 IMM UV oil-immersion objective (resulting voxel size: $0.7568 \mu \mathrm{m} \times 0.7568 \mu \mathrm{m} \times 0.6294 \mu \mathrm{m})$ or with a HCX PL APO lambda blue $63.0 \times 1.40$ OIL UV oil-immersion objective (resulting voxel size: $0.2403 \mu \mathrm{m} \times 0.2403 \mu \mathrm{m} \times 0.9651 \mu \mathrm{m}$ ).

\section{Image Data Analysis}

Confocal stacks were analyzed with Image $^{1}$ and the following installed plug-ins: Biovoxxel extensions (Jan Brocher), BioFormats extension (LOCI), Cell Counter Plug-in (Kurt de Vos). Z-stacks of every 2 nd horizontal brain section were made and the slices in each $\mathrm{z}$-stack were summed into $\mathrm{z}$-projections. To derive a mask encompassing the area of rAAV transduction a gaussian blur $(r=20 \mu \mathrm{m})$ filter was applied to the thresholded z-projection of the EYFP channel (threshold algorithm: mean; threshold value: $5 \mathrm{x}$ background noise intensity in the EYFP channel). This image was then converted into a binary mask and the mask saved as region of interest (ROI) in ImageJ. The EYFP sum z-projection was overlaid with the ROI now encompassing the transduction area. The transduction area (=area within ROI) was measured using the standard built-in tools in ImageJ. Total neurons (neuronal marker) and transduced neurons (EYFP) were manually counted inside the transduction area with the help of the Cell Counter plug-in.

\section{In Vitro Electrophysiology}

Gerbils (10-28 days post injection) were anesthetized with Isofluran (IsoFlo, Abbot Laboratories) and decapitated. Brains were removed and kept in slice solution (50 mM sucrose, $25 \mathrm{mM}$ $\mathrm{NaCl}, 25 \mathrm{mM} \mathrm{NaHCO}, 2.5 \mathrm{mM} \mathrm{KCl}, 1.25 \mathrm{mM} \mathrm{NaH} \mathrm{PO}_{4}$, $3 \mathrm{mM} \mathrm{MgCl}_{2}, 0.1 \mathrm{mM} \mathrm{CaCl}_{2}, 25 \mathrm{mM}$ glucose, $0.4 \mathrm{mM}$ ascorbic acid, $3 \mathrm{mM}$ myo-inositol and $2 \mathrm{mM}$ sodium pyruvate; SigmaAldrich Chemie $\mathrm{GmbH}$ ) bubbled with $95 \% \mathrm{O}_{2}$ and $5 \% \mathrm{CO}_{2}$. Horizontal brainstem sections of $200 \mu \mathrm{m}$ thickness were cut using a VT1000S or VT1200S vibratome (Leica Biosystems) and incubated in extracellular recording solution $\left(25 \mathrm{mM} \mathrm{NaHCO}_{3}\right.$, $2.5 \mathrm{mM} \mathrm{KCl}, 1.25 \mathrm{mM} \mathrm{NaH} \mathrm{PO}_{4}, 125 \mathrm{mM} \mathrm{NaCl}, 25 \mathrm{mM}$ glucose, $0.4 \mathrm{mM}$ ascorbic acid, $3 \mathrm{mM}$ myo-inositol, $2 \mathrm{mM}$ sodium pyruvate, $2 \mathrm{mM} \mathrm{CaCl}_{2}$, and $1 \mathrm{mM} \mathrm{MgCl}_{2}$; Sigma-Aldrich Chemie $\mathrm{GmbH}$ ) at $36^{\circ} \mathrm{C}$ for $45 \mathrm{~min}$, bubbled with $5 \% \mathrm{CO}_{2}$ and $95 \% \mathrm{O}_{2}$.

Brain slices were transferred to a recording chamber attached to a microscope (Olympus BX51WI Microscope, Olympus Plan N $4 \times / 0.10$ Objective, Olympus LUMPlanFL N 60×/1.00 W Objective, Olympus TH4-200 infrared lamp, Olympus Aplanat Achromat 1.4 NA Oil Condenser and LEJ LQ-HXP-120-3 fluorescence lamp) and continuously perfused with extracellular solution containing $50 \mu \mathrm{M}$ D-AP5 [D-(-)-2amino-5-phosphonopentanoic acid] and $20 \mu \mathrm{M}$ DNQX (6,7dinitroquinoxaline-2,3-dione; Biotrend) to block glutamatergic transmission. Voltage-clamp recordings were carried out at near-physiological temperature $\left(33-36^{\circ} \mathrm{C}\right)$ and current-clamp recordings at room temperature. Cells were visualized and imaged with an Orca R2 CCD camera (Hamamatsu). Voltageand current-clamp recordings were performed with an EPC9 and EPC10 triple-patch amplifier (HEKA Elektronik). For voltageclamp recordings access resistance was compensated to residual of 3-4 M $\Omega$ of the initial value; data was acquired at a sample rate of $20 \mathrm{kHz}$ and filtered at $2.9 \mathrm{kHz}$. For whole cell voltage-clamp recordings an internal solution ( $\mathrm{pH} 7.2)$ consisting of $105 \mathrm{mM}$ Cs gluconate, $26.7 \mathrm{mM} \mathrm{CsCl}, 10 \mathrm{mM}$ HEPES, $20 \mathrm{mM}$ TEA-Cl,

\footnotetext{
${ }^{1}$ http://fiji.sc/Fiji
} 
$5 \mathrm{mM}$ Cs-EGTA, $3.3 \mathrm{mM} \mathrm{MgCl}_{2}, 2 \mathrm{mM} \mathrm{Na}_{2}$-ATP, $0.3 \mathrm{mM} \mathrm{Na}_{2}$ GTP, and $3 \mathrm{mM} \mathrm{Na}$-phosphocreatine was used. For whole cell current-clamp recordings the internal recording solution $(\mathrm{pH}$ 7.2) consisted of $145 \mathrm{mM} \mathrm{K}$-gluconate, $5 \mathrm{mM} \mathrm{KCl}, 15 \mathrm{mM}$

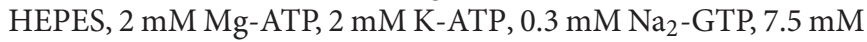
$\mathrm{Na}_{2}$-phosphocreatine, and $5 \mathrm{mM} \mathrm{K-EGTA.} \mathrm{Transduced} \mathrm{neurons}$ were visually identified by their fluorescence (CatCH-mCherry or NpHR3.0-EYFP) and accessed with glass pipettes (resistance between 3 and $5 \mathrm{M} \Omega$ ). Light stimulation was delivered either by a KSL 083 (590 nm) LED or a KSL 010 (470 nm) LED controlled by a KSL Duo LED controller (Rapp OptoElectronic).

\section{Analysis of in Vitro Data}

Electrophysiological data were analyzed with IGOR Pro (WaveMetrics) with the Patcher's Power Tools 2.19 extension ${ }^{2}$, Excel 2013 (Microsoft), Graphpad Prism 5 (Graphpad Software) and Sigmaplot 11.0 (Systat Software). Latencies were measured as the time from trigger onset to AP peak; only APs that appeared within $30 \mathrm{~ms}$ after trigger onset were considered successful. Vector strength $(r)$ was calculated using the following formula (Goldberg and Brown, 1969):

$$
r=\frac{\sqrt{\left(\sum \cos \theta_{\mathrm{i}}\right)^{2}+\left(\sum \sin \theta_{\mathrm{i}}\right)^{2}}}{n}
$$

The jitter is equal to the standard deviation $(S D)$ of AP latencies calculated according to the following formula:

$$
\text { Jitter }=s d=\sqrt{\frac{\sum(\mathrm{x}-\overline{\mathrm{x}})^{2}}{(\mathrm{n}-1)}}
$$

\section{In Vivo Electrophysiology}

\section{Surgical Procedures}

Three to 4 weeks after injection of AAV8(Y733F), gerbils were anesthetized with an initial intraperitoneal injection ( $0.5 \mathrm{ml} / 100 \mathrm{~g}$ body weight) of a physiological $\mathrm{NaCl}$ solution (Ringer's solution) containing $20 \%(\mathrm{v} / \mathrm{v})$ ketamine chloride (Ketavet, Zoetis Deutschland $\mathrm{GmbH}$ ) and 2\% (v/v) xylazine chloride (Rompun, Bayer HealthCare AG). Depth of anesthesia was monitored by testing the hind leg withdrawal reflex. During recording, anesthesia was maintained at a rate of $2.4 \mu \mathrm{l}$ per $100 \mathrm{~g}$ body weight per minute by means of an automatic pump (801 Syringe Pump; Univentor). Animal body temperature was monitored and maintained using a thermostatically controlled heating pad (Fine Science Tools). Skin and tissue covering the upper part of the skull were removed carefully and a small metal rod was mounted on the frontal part of the skull using UVsensitive dental-restorative material (Charisma, Heraeus Kulzer). The tragus on both ears was incised to ease access to the ear canal, and headphones (Etymotic Research ER4-PT) were placed into the ear canals. Thereafter, the gerbil was transferred into a soundattenuated chamber and the animal's head was fixed in a custommade stereotaxic device (Schuller et al., 1986). The animal's head position in the recording chamber was aligned by stereotactic landmarks on the surface of the skull (Loskota et al., 1974).

${ }^{2}$ https://www.wavemetrics.com/users/tools_aux001.htm
To enable electrodes to penetrate into the DNLL, a craniotomy and durotomy was performed behind the sinus transversus lateral to midline. During the recording session, Ringer's solution was applied to the opening to prevent dehydration of the brain. We labeled the last recording site by a current-induced lesion (25 $\mu \mathrm{A}, 90 \mathrm{~s}$ ) using metal electrodes (WPI Stimulus Isolator A360). Finally, the animal was killed by intraperitoneal injection of pentobarbital as described above.

\section{Light Stimulation}

An optic fiber (Thorlabs Multimode Optical Fiber, 0.39 NA, $\varnothing=200 \mu \mathrm{m}$ Core, HighOH) was inserted into a cannula with the upper end glued into a ceramic ferrule (Thorlabs FC/SC Ceramic Ferrule, $\varnothing=2.5 \mathrm{~mm}$, multimode, $230 \mu \mathrm{m}$ hole- $\varnothing$ ). The fiber was then connected to the LED light source (Thorlabs Fiber Coupled High Power LED, Blue-470 nm or Orange-617 nm, SMA, $1000 \mathrm{~mA}$ ) via $2 \mathrm{~m}$ of connectorized optic fiber and mating sleeve (Thorlabs Ceramic Split Mating Sleeve for $\varnothing=2.5 \mathrm{~mm}$ Ferrules). LEDs were controlled with a 4Channel LED Driver (Thorlabs) and Single LED Connector Hub (Thorlabs) and triggered and intensity-adjusted with a patchclamp EPC10 amplifier (HEKA). The cannula with the fiber was mounted on a micromanipulator (IVM single axis, Scientifica) and placed either within 100-350 $\mu \mathrm{m}$ proximity of the recording site or exactly contralateral to it.

\section{Extracellular Single-Cell Recording}

We recorded single-unit responses utilizing glass electrodes filled with $1 \mathrm{M} \mathrm{NaCl}(\sim 7-10 \mathrm{M} \Omega)$. The electrodes were maneuvered by a single-axis micromanipulator (Scientifica). Extracellular APs were recorded with a patch-clamp amplifier (EPC10; HEKA) and converted by a RZ-6 Multi I/O Processor (Tucker-Davis Technologies). Residual line noise was removed with a noise eliminator (Humbug, Quest Scientific). AP isolation (signal-tonoise ratio $>3$ ) was performed online by visual choice (AP amplitude) and offline by spike cluster analysis based on stable peak amplitudes and spike waveforms (Brainware, Jan Schnupp, TDT).

\section{Acoustic Stimulation}

Frequency responses were calibrated for the headphones. Acoustic stimuli were generated digitally, converted to an analog signal at $\sim 200 \mathrm{kHz}$ sampling rate (RZ-6 Multi I/O Processor, Tucker-Davis Technologies), attenuated and conveyed to the headphones (ER4-PT, Etymotic Research). The standard search stimulus presented to the contralateral ear was a broadband stimulus (white noise bursts) with a duration of $200 \mathrm{~ms}$ and squared-cosine rise/fall times of $5 \mathrm{~ms}$. The same stimulus with a duration of $20 \mathrm{~ms}$ was presented on the ipsilateral ear after the first $100 \mathrm{~ms}$. The inter-stimulus interval for each repetition was $900 \mathrm{~ms}$. For all recordings, stimulus presentation was pseudo-randomized. Acoustically evoked responses were searched for by administering binaurally delivered noise stimuli without interaural time and intensity differences. A neuron's best frequency $(\mathrm{BF})$ and threshold were determined by means of binaurally identical (interaural intensity difference/interaural time difference $=0$ ) sinus tone stimulation. BF was defined as the 
frequency that elicited responses at the lowest sound intensity. Threshold was assigned to the lowest sound intensity evoking a noticeable response at BF. All stimuli applied in the course of our experiments were based upon these characteristics.

\section{Statistical Analysis}

All data are given as either original measurements or mean/median. Statistical analysis was performed using Prism 7 (GraphPad Software, Inc., La Jolla, CA, United States). The statistical tests applied are mentioned in the Figure legends.

\section{RESULTS}

\section{AAV8(Y733F).hSyn Enables Neuron-Specific Expression of Transgenes in the Gerbil Brainstem and Midbrain}

The capacity of AAV8(Y733F) to stably and specifically transduce neurons in the gerbil was tested by stereotactically injecting $250 \mathrm{nl}$ of the control vector AAV8(Y733F).hSyn.EYFP, which expresses EYFP under the control of the hSyn promoter, into three different nuclei of the auditory system (IC, DNLL, and MNTB) in anesthetized animals (P 28-60) (Keplinger, 2016). After the indicated expression periods, animals were sacrificed by administering a lethal dose of pentobarbital (IC: 7, 14, 21, and 28 dpi; DNLL: 28 dpi; MNTB: $21 \mathrm{dpi}$ ). In all three nuclei, expression of the control fluorophore EYFP was found in coronal sections (50 $\mu \mathrm{m}$ thick) of fixed brains (Figure 1). A 100\% success rate was achieved in the IC $(n=12)$ and about one-third of injections in the DNLL and MNTB were correctly targeted. The area of transduction was always detected focally around the injection site (IC, $n=12 / 12$; $\mathrm{MNTB}, n=3 / 3$; DNLL, $n=3 / 3$ ) and no evidence for anterograde or retrograde transport was observed. Additional expression of EYFP was detected in the ECIC $(n=3 / 12)$ upon injection into the lateral part of the central IC (CIC), and along the injection tract when virus particles were injected into the MNTB $(n=1 / 3)$ or into the DNLL $(n=1 / 3)$ (data not shown). All transduced, EYFP-expressing cells showed co-labeling with the neuronal markers Map2 and/or NeuN. As a negative control, a glial scar caused by bolus injection of $250 \mathrm{nl}$ of AAV8(Y733F).hSyn.EYFP into the IC displayed extensive labeling of the S100B marker specific for astrocytes at $14 \mathrm{dpi}$, but no EYFP expression was detected in these cells (Supplementary Figure S1).

\section{Efficiency of AAV8(Y733F).hSyn.EYFP Transduction and Expression}

We constantly observed that not all neurons within the transduction area were transduced. For instance, about half of MNTB neurons within the target area were transduced by a single virus injection of $250 \mathrm{nl}(n=2)$. Thus, the transduction efficiency was evaluated by quantification of the number/fraction of transduced neurons, the size of the transduction area and the signal-to-noise ratio (SNR) (Figure 2). AAV8(Y733F).hSyn.EYFP was injected into the IC of three animals (P28-P49) for each expression period tested $(1,2,3$, and 4 weeks). At least five coronal sections (50 $\mu \mathrm{m}$ thickness) per animal were immunohistochemically labeled NeuN (Figure 2A). The IC was chosen since we observed a positive expression result in 100\% of injections. EYFP expression was already detectable 1 week after injection. We counted transduced and total neurons in the transduction area by analyzing the sum projection of the confocal $\mathrm{z}$-stacks (Figure 2C). Including all time points, we manually counted 17579 transduced neurons in a total sample of 32369. At all four time points, roughly half of the neurons within the injection area (average over all time points, 54\%) were transduced (Figure 2D). The size of the transduction area was determined by thresholding the mean intensity for each section (Figure 2B). None of these parameters changed significantly with the expression time.

To quantify expression levels in transduced cells over time, the SNR was measured and expressed relative to the background fluorescence ("fold background"; Figure 2E). For each biological replicate the average EYFP intensity of 52 cells was measured in the sum projection of a confocal z-stack. The SNR was found to increase over time, with the highest values and highest dynamics being recorded at 4 weeks after injection. In summary, longer expression times resulted in significantly increased expression levels in individual cells (at 4 weeks 48 times higher than at 1 week after injection), but not in significantly greater numbers or fractions of transduced neurons.

\section{Optical Modification of Neuronal Activity in Acute Gerbil Brain Slices}

We performed whole-cell patch-clamp recordings in both current-clamp and voltage-clamp modes to assess the ability of the channelrhodopsin/halorhodopsin to excite/inhibit transduced neurons in response to light. By measuring the photostimulated responses in acute brain slices, we confirmed the functional expression of the transgenes in the plasma membrane. We then characterized the properties of the optogenetic tools as a function of the biophysical properties of the neurons transduced, by determining the levels of functional transgene expression and the effectiveness of the optical stimulation. Animals (P2-P30) were injected with AAV8(Y733F) vectors and sacrificed after 10-28 dpi for preparation of coronal sections of $150-200 \mu \mathrm{m}$ thickness. Glutamatergic transmission in the slices was blocked by means of D-AP5 and DNQX.

\section{Light-Induced Generation of APs in IC and MNTB Neurons Expressing CatCH}

The AAV8(Y733F).hSyn.CatCH.mCherry vector was injected into the IC (Figure 3A) and into the MNTB (Figure 3D), and transduced neurons were identified in brain slices based on their mCherry fluorescence (Supplementary Figure S2). The fluorescence was distributed in a spotty pattern pointing at expression in the plasma membrane (Figures 3A,D). Accordingly, $470 \mathrm{~nm}$ light pulses elicited APs in 11 out of 21 recorded cells after $10 \mathrm{dpi}$ (Figures 3B,E). The remaining neurons were responsive to light, but even after 28 dpi only subthreshold depolarizations were recorded (Figures 3C,F). As APs could be evoked by current injections in these latter neurons (data 


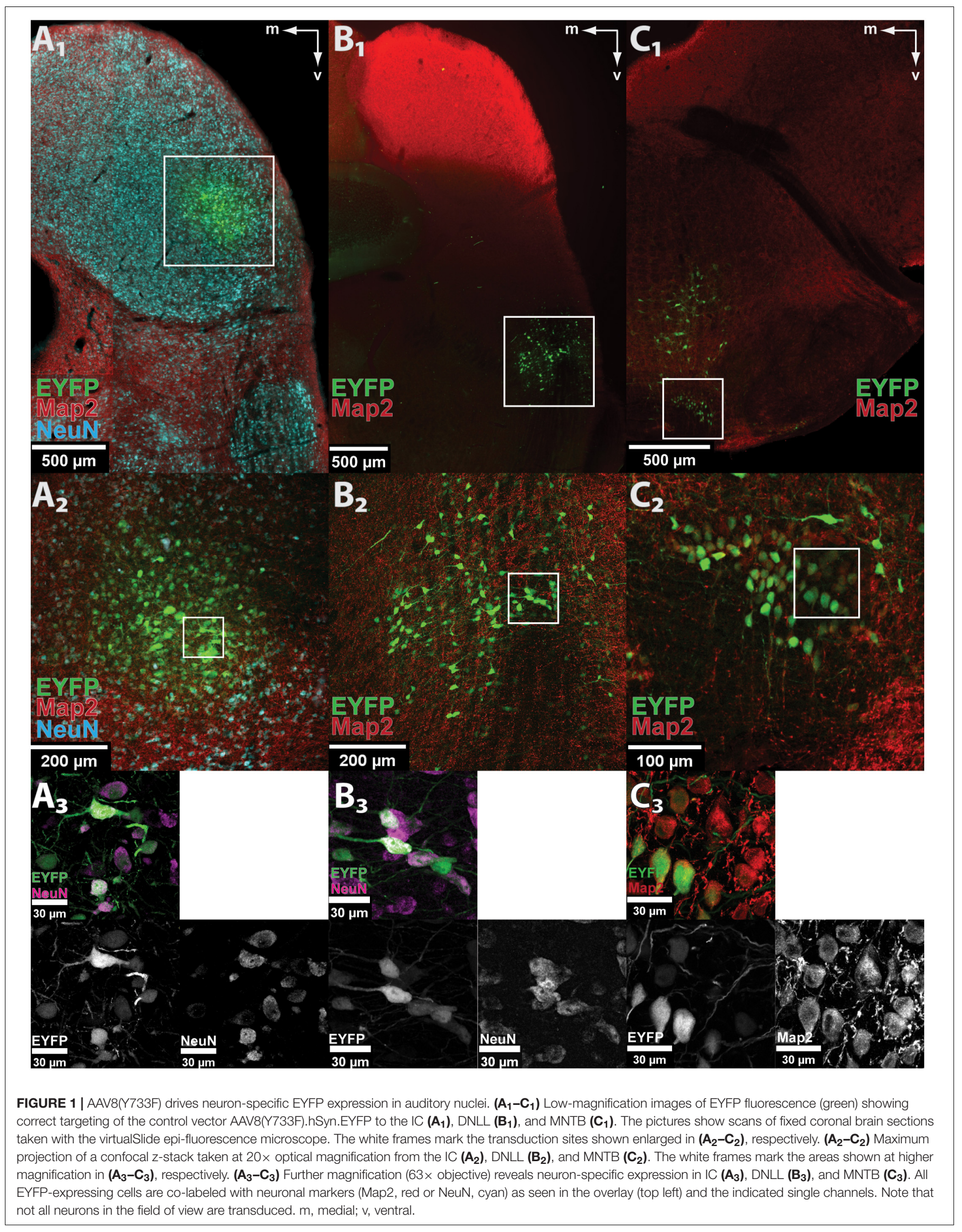




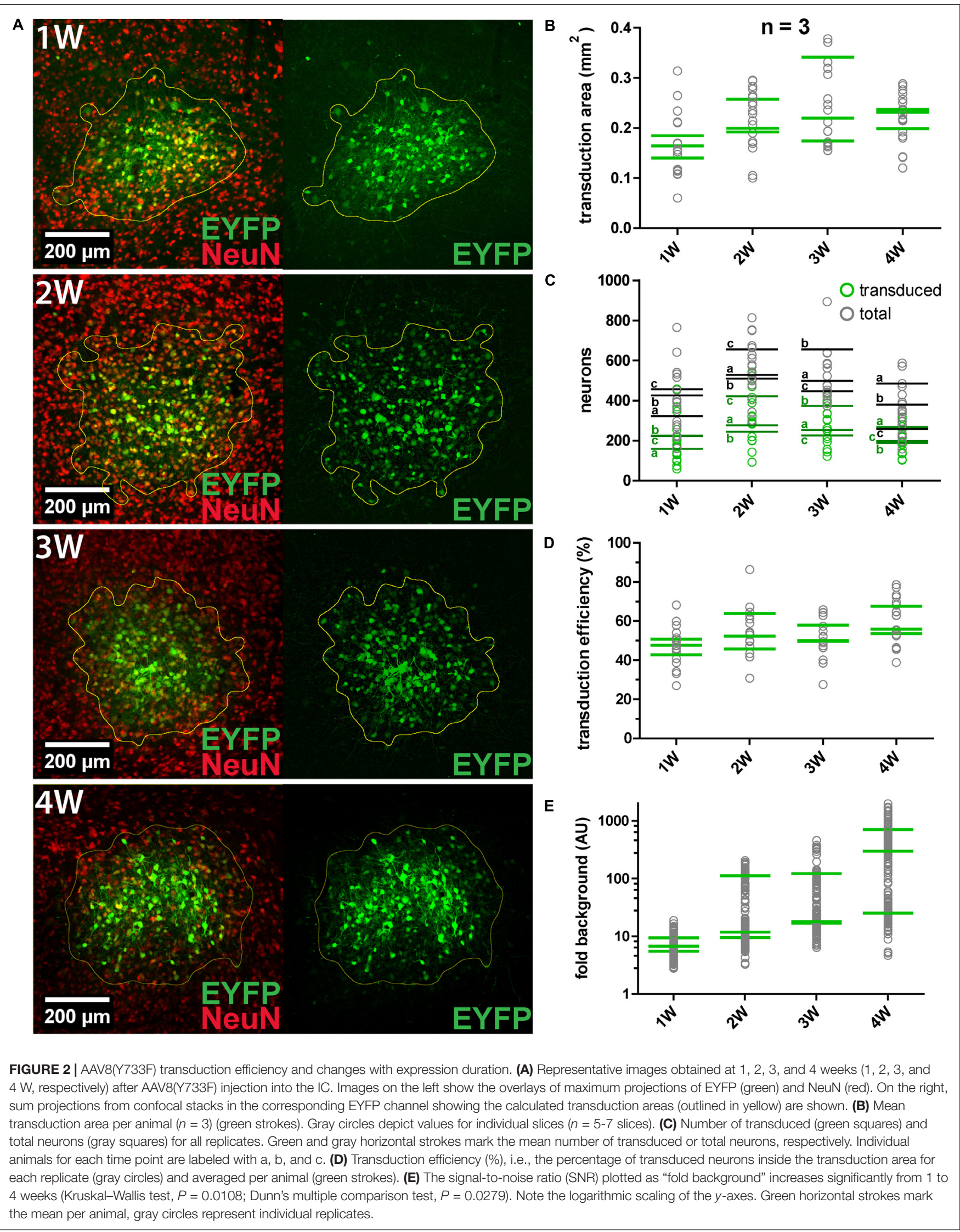




\section{A}

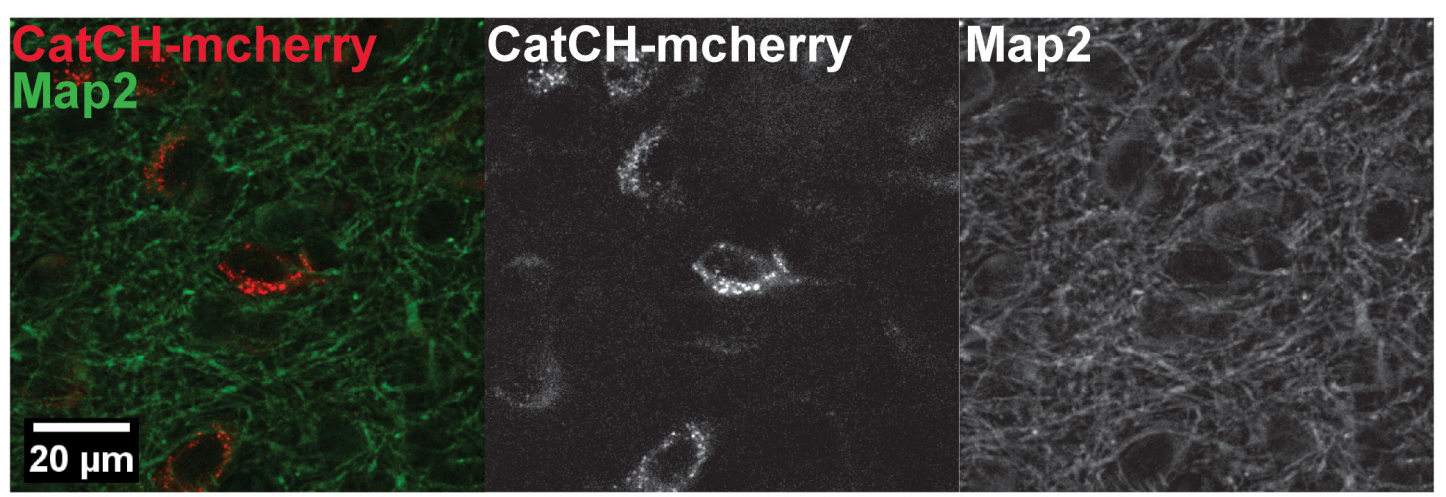

B

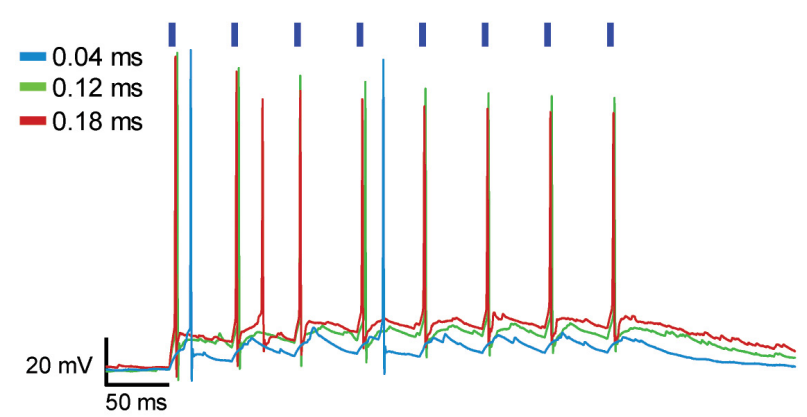

C

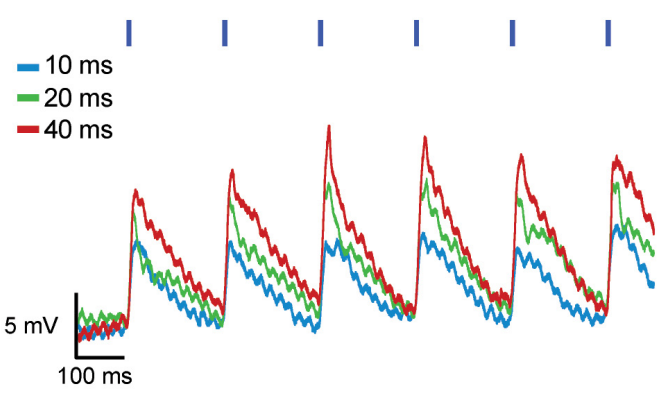

D

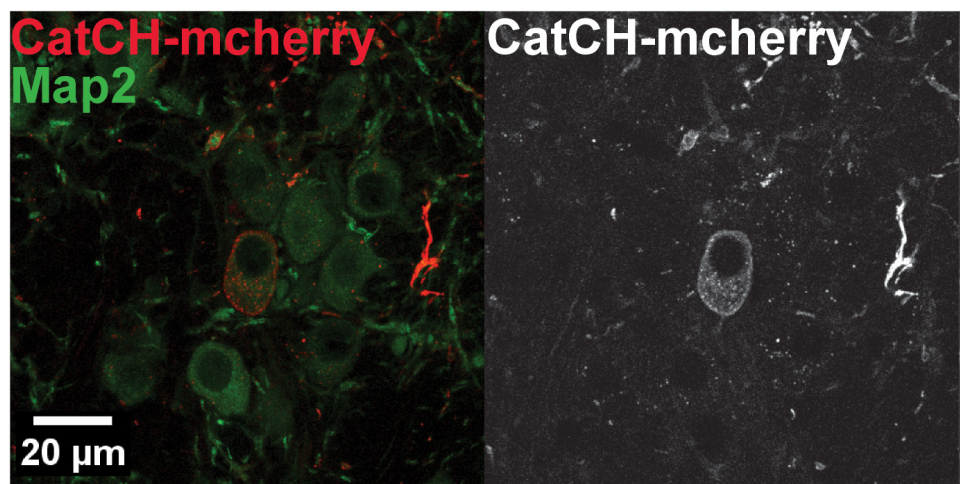

\section{Map2}

E | | | | | | | | | |

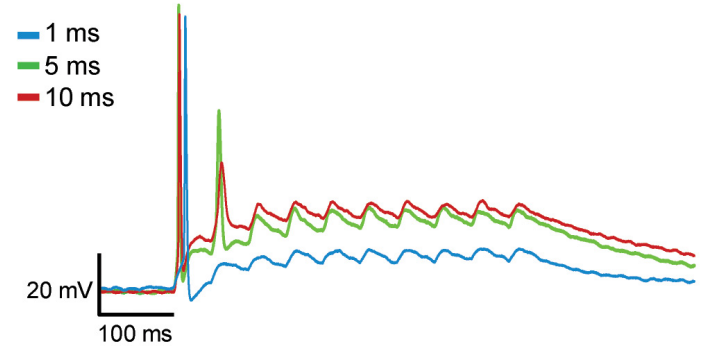

$\mathbf{F}$

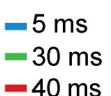

$-40 \mathrm{~ms}$

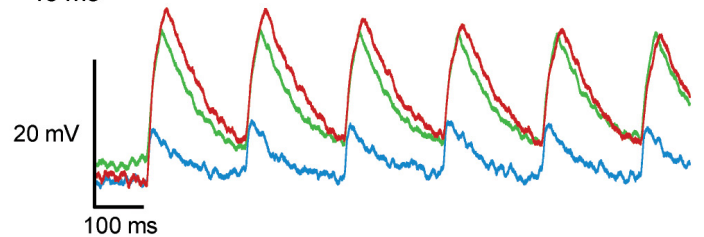

FIGURE 3 | Transduced IC and MNTB neurons expressing CatCH-mCherry can be depolarized with blue light. (A) The maximum projections from a confocal z-stack show transduced IC neurons expressing CatCH-mCherry (red). Green fluorescence reveals the Map2 marker. (B) Transduced IC neurons fire when stimulated with light pulses of varying pulse widths ( $40 \mu \mathrm{s}$, blue trace; $120 \mu \mathrm{s}$, green trace, $180 \mu \mathrm{s}$, red trace) at $20 \mathrm{~Hz}$. (C) Voltage traces of an IC neuron photostimulated at $5 \mathrm{~Hz}$ with pulses of varying durations (10 ms, blue trace; $20 \mathrm{~ms}$, green trace; $40 \mathrm{~ms}$, red trace) show only sub-threshold responses. (D) Maximum projections from a confocal z-stack depict transduced MNTB neurons expressing CatCH-mCherry (red). Green labeling represents immunostaining of Map2. (E) Transduced MNTB neurons fire when stimulated with light pulses of varying pulse widths (1 ms, blue trace; $5 \mathrm{~ms}$, green trace; $10 \mathrm{~ms}$, red trace) at $20 \mathrm{~Hz}$. (F) Voltage traces of a MNTB neuron stimulated at $5 \mathrm{~Hz}$ with light pulses of different widths $(5 \mathrm{~ms}$, blue trace; $30 \mathrm{~ms}$, green trace; $40 \mathrm{~ms}$, red trace) show only sub-threshold responses. (B,C,E,F) Blue strokes above voltage traces indicate the number and onset timing of the light pulses. Width of blue strokes is not to scale but enlarged for better visibility. 
not shown), we assume that expression levels of $\mathrm{CatCH}$ were too low for optical activation.

As crucial prerequisite for in vivo utilization of $\mathrm{CatCH}$ in neuron populations of the gerbil brain, we examined the dependency of $\mathrm{CatCH}$ responses on the properties of the light pulse (optical stimulation frequency, 5-100 Hz; lightpulse widths, $40 \mu \mathrm{s}-95 \mathrm{~ms}$ ) and neuronal biophysics (input resistance). Optical excitation was considered to be effective when each light pulse elicited one AP. Any additional evoked spike was counted as an extra spike. The temporal precision of the cellular responses upon effective light stimulation was also quantified. Frequently, not all stimulation protocols and combinations of light-pulse widths and frequencies could be applied to every cell, as some did not survive for long enough.

We analyzed the first spike of the train because of the absence of any spike history from preceding spikes (Figures $4 \mathbf{A}, \mathbf{E}$ ). Longer light pulses were more efficient, but also evoked extra spikes with a higher probability (Figures $\mathbf{4 B}, \mathbf{F}$ ). The shortest achievable spike latencies varied between individual cells. Usually, latencies became shorter with increasing light pulse duration and vice versa (Figures 4C,G). To quantify temporal fidelity, the level of jitter of photo-stimulated spikes was determined, and found to be in the (sub-) millisecond range (0.14-9.8 $\mathrm{ms})$. In general, it decreased with increasing pulse duration (Figures $\mathbf{4 D}, \mathbf{H}$ ). The latency of the first spike did not differ significantly from those of the succeeding spikes and its mean value was $12.9 \mathrm{~ms} \pm 6.6 \mathrm{~ms}(S D ; n=9)$ for the first one and $14.0 \mathrm{~ms} \pm 7.2 \mathrm{~ms}(S D ; n=10)$ for the subsequent spikes (Figures $4 \mathbf{I}, \mathbf{J}$ ). The shortest latency of the first spikes measured for all conditions was $2.3 \mathrm{~ms}$ (20ms light pulse at $5 \mathrm{~Hz}$ ) and the longest was $32.7 \mathrm{~ms}$ (5-ms light pulse at $10 \mathrm{~Hz}$ ). The latency was independent of the membrane resistance (Figure $4 \mathbf{K}$ ). We calculated the vector strength $r$ as another measure of temporal precision for a range of frequencies and pulse widths, in order to identify possible resonance effects that would allow more precise spiking at certain stimulation frequencies. Assuming that resonance is present, stimulation at the resonance frequency would be expected to be more efficient. However, no facilitation by resonance was observed and light-pulse duration turned out to be the dominant factor determining temporal precision (data not shown).

\section{Light-Induced Inhibitory Currents and Suppression of APs in IC and MNTB Neurons Expressing NpHR3.0}

In order to hyperpolarize transduced cells with light and thereby suppress APs, AAV8(Y733F).hSyn.NpHR.EYFP was injected into the IC and MNTB. Experimental conditions were similar to those described above. Transduced neurons were identified for electrophysiological recordings by their EYFP fluorescence. NpHR3.0 was activated with orange light pulses $(617 \mathrm{~nm})$ of $500 \mathrm{~ms}$ duration. The membrane potential was recorded in the current-clamp mode (Figure 5) and photocurrents were examined in the voltage-clamp mode (Figure 6).

Neurons were stimulated with a train $(10 \mathrm{~Hz})$ of current injections each 10\% above AP threshold, and light pulses of varying duration were simultaneously presented to suppress spikes (Figure 5). At $10 \mathrm{dpi} \mathrm{NpHR}$ expression levels were already sufficiently high to inhibit spiking. When the light pulse did not overlap the current stimulation, suppression was ineffective (Figure 5A). Photosuppression of individual spikes was successful in only one out of the five neurons tested, and less efficient than the suppression of spike trains by longer light pulses. In most neurons, efficient photosuppression was achieved with light pulses whose onset preceded the current injection by several ms and outlasted it by the same margin (Figure 5B). Postinhibitory rebound spikes due to light-induced hyperpolarization were frequently observed (Figures 5C,D).

The elicited photocurrent exhibited a strong dependency on light intensity and increasing the light power resulted in its saturation (Figures 6A,D). A decrease in efficiency was observed during stimulation (Figures 6A,B), due to depletion of 11-cisretinal by photoconversion. The maximum elicited photocurrent in IC $(n=15)$ and MNTB neurons $(n=8)$ varied widely from cell to cell (Figure 6B). The mean peak photocurrent attained at the maximum light power used $\left(6.2 \mathrm{~mW} / \mathrm{mm}^{2}\right.$; Figure $\left.6 \mathrm{~B}\right)$ was $390 \mathrm{pA} \pm 424 \mathrm{pA}(S D)$ and the mean steady-state value was $344 \mathrm{pA} \pm 408 \mathrm{pA}(S D)$. A slight decrease in photocurrent was observed for successive sweeps, but this quickly leveled off (Figure 6C).

The kinetics of photocurrent induction were analyzed by calculating time constants for $\mathrm{ON}\left(\mathrm{tau}_{\mathrm{on}}\right)$ and OFF reactions $\left(\mathrm{tau}_{\mathrm{off}}\right)$ based on exponential fits (Figures 6E,F). The rise time $\left(t_{20 / 80}\right)$ for an increase in the peak photocurrent from 20 to $80 \%$ of its maximum was determined as well, since noise perturbations at $50 \mathrm{~Hz}$ had occurred in some of the recordings, and $t_{20 / 80}$ was less affected by this noise. For the maximal light power, the mean values obtained were: $\operatorname{tau}_{\mathrm{on}}=1.7 \mathrm{~ms} \pm 0.6 \mathrm{~ms}(S D)$, $t_{20 / 80}=2.2 \mathrm{~ms} \pm 1 \mathrm{~ms}(S D)$, and $\mathrm{tau}_{\text {off }}=2.4 \mathrm{~ms} \pm 0.8 \mathrm{~ms}(S D)$ (Figure 6E). These kinetic parameters were independent of the intensity of the light pulse (Figure 6F).

\section{NpHR3.0-Mediated Photosuppression Efficiently Modifies Acoustically Evoked Responses in the IC and the DNLL of Anesthetized Gerbils}

We then recorded extracellular potentials of NpHR3.0-expressing neurons in the IC and the DNLL of anesthetized gerbils while simultaneously presenting broadband noise stimuli and pulsed light. Animals were stereotactically injected on P28 or later with AAV8(Y733F).hSyn.CatCH.mCherry.T2A.NpHR3.0 or AAV8(Y733F).hSyn.NpHR3.0.EYFP. The DNLL was chosen as the primary experimental target for in vivo optogenetic experiments in order to elucidate its role in the phenomenon of precedence, as outlined in the section "Introduction." The IC was targeted because of its physical size, its accessibility (short insertion distances for both virus injection and optical fiber) and because the firing patterns of its neurons upon auditory stimulation have been well characterized. These experiments were based on parameters (e.g., light-pulse width and timing) determined for effective optical silencing with NpHR 3.0 in our patch-clamp experiments on acute brain slices. We first set up light delivery into the transduced area and then collected in vivo 


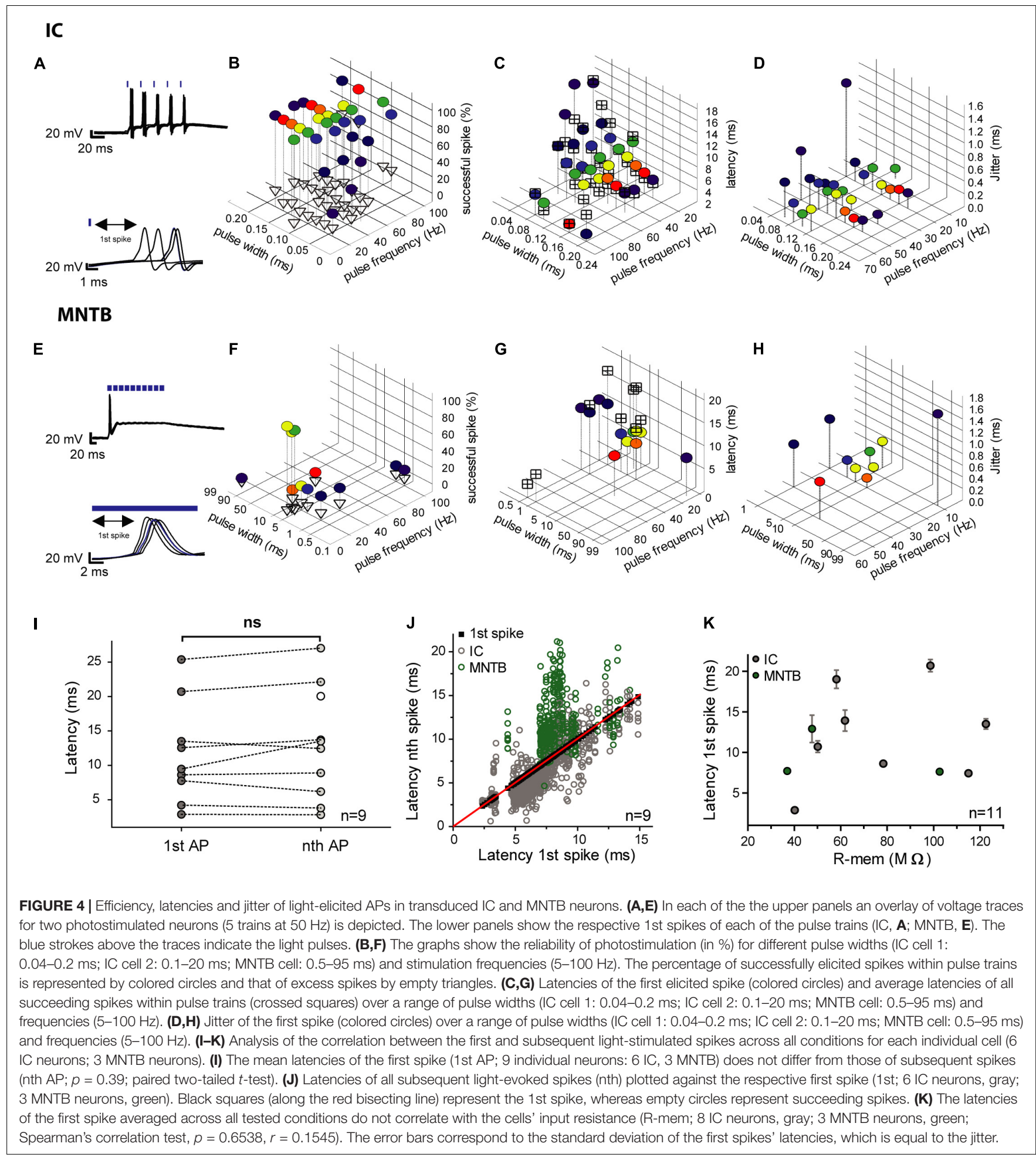

evidence for the impact of light on the firing behavior of stimulusresponsive neurons. Technically, injection of the rAAV vectors, insertion of the optical fiber and positioning of the recording electrode are more demanding for the DNLL than for the IC. Expression times of at least 4 weeks were chosen in order to obtain high expression levels of NpHR3.0. We maximized the volume containing transduced neurons by administering three consecutive virus injections (accepting a slightly increased probability of off-target transduction). The fluorescence emitted by the expressed NpHR3.0-EYFP was concentrated at the plasma membrane and detectable in axons and dendrites of transduced neurons (Figures 5E-G). 

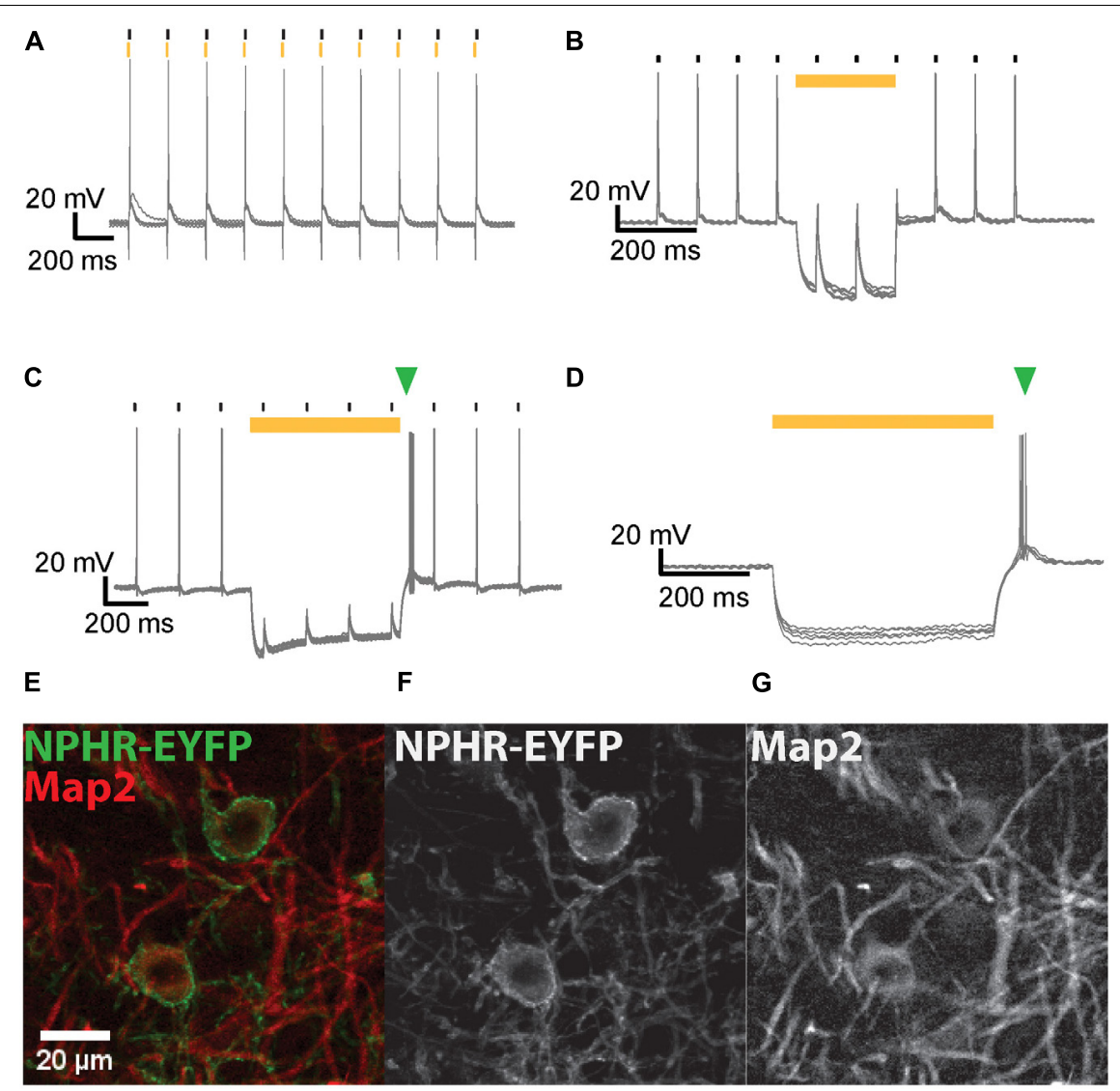

FIGURE 5 | NpHR3.0-mediated suppression of AP generation in IC and MNTB neurons. (A) Overlay of voltage traces for an IC neuron expressing NpHR3.0-EYFP when stimulated with current injections (black strokes) immediately after 5-ms light pulses (yellow strokes) applied at $10 \mathrm{~Hz}$. The neuron generates an AP upon current injection despite its light-evoked hyperpolarization. (B) A 200-ms light pulse (yellow bar) effectively suppresses spiking of IC neurons in response to current injections (black strokes) at $10 \mathrm{~Hz}$. (C) AP generation induced by current injection (black strokes) in a transduced MNTB neuron is suppressed by a 250 ms light pulse (yellow bar). An extra spike (green triangle) is evoked after termination of the light pulse. (D) A 500-ms light pulse (yellow bar) induces hyperpolarization of an IC neuron expressing NpHR3.0-EYFP, but the neuron promptly spikes at the end of the light pulse (green triangle). (E) Exemplary DNLL neurons exhibit co-localization of NpHR3.0-EYFP (green) and Map2 (red). The image represents the sum projection of a confocal z-stack imaged with the 63x objective. The individual channels for NpHR3.0-EYFP (F) and Map2 (G) are shown as well.

The sensory evoked spiking activity of transduced cells in the DNLL $(n=2)$ and IC $(n=4)$ could be suppressed by light (Figure 7). To quantify the level of photosuppression, spike counts during light application were normalized to the control condition. In one DNLL neuron, spike counts were reduced by $98 \%$ while auditory stimulation (mono- as well as binaural) was ongoing, and actually preceded the light pulse by $40 \mathrm{~ms}$ (Figure 7A). In a recording from an EE neuron in the DNLL, a light pulse that preceded the (mono- as well as binaural) auditory stimulation by $9 \mathrm{~ms}$ suppressed spiking activity by $63 \%$ (Figure 7B). After the light pulse, spiking activity increased to control spike rates. The extent of photosuppression varied from cell to cell (1-98\%; Figures 7D,E), since distances from the tip of the optical fiber and expression levels of NpHR3.0 itself also varied. The two DNLL neurons (Figures 7A,B) were suppressed more effectively than the recorded IC neurons (Figure 7C). The effect size represents the difference in spike numbers between control and light conditions (normalized to the control condition) during the entire auditory stimulation period (see "Materials and Methods" section). The mean effect size was higher for monaural auditory stimulations (E0, 0.42; $0 \mathrm{E} / 0 \mathrm{I}, 0.65)$, whereas for binaural stimulation the mean effect size was 0.38 (EE/EI) (Figure 7F). Owing to the method used to calculate effect sizes, these were smaller for light pulses shorter than the auditory stimulation period. The latencies of the first auditory evoked spike were affected in only one of the recordings (Figure 7G).

\section{DISCUSSION}

\section{AAV8(Y733F) Is a Versatile Tool for Neuron-Specific Transduction in the Gerbil}

Using AAV8(Y733F) vectors bearing the relatively short hSyn I promoter, we were able to drive neuron-specific transgene expression in the MNTB, DNLL, and IC of Mongolian gerbils, 

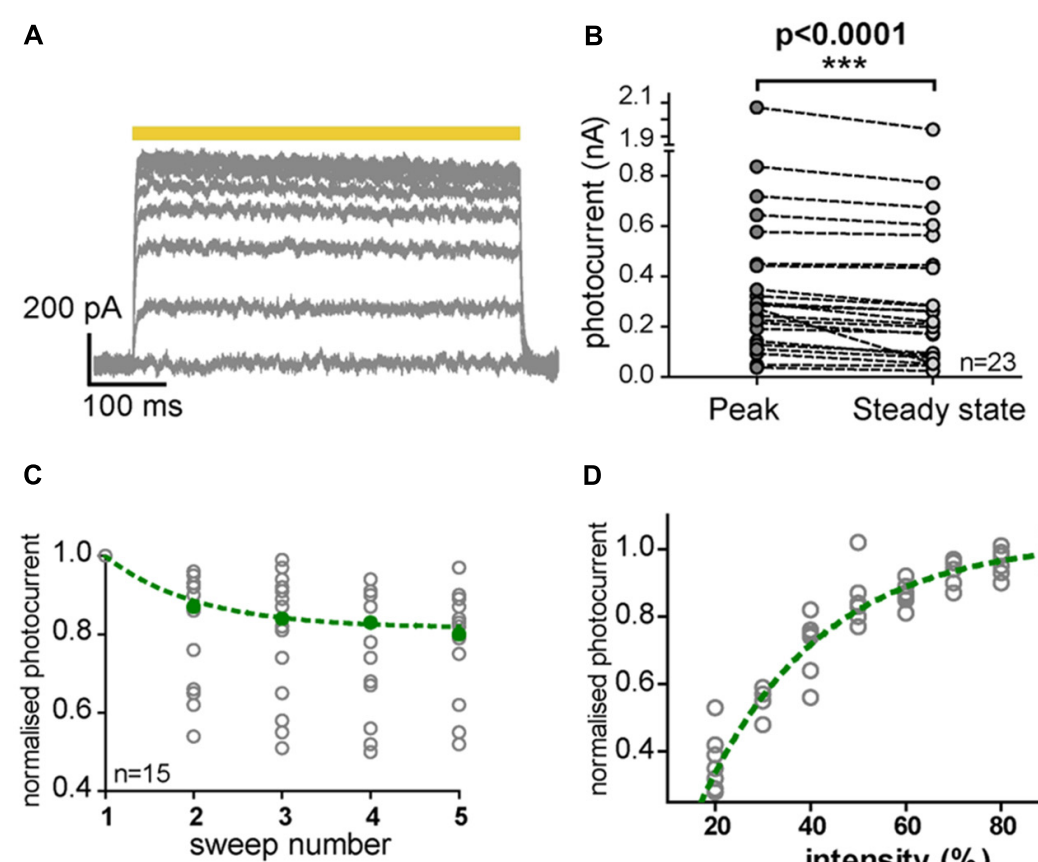

D
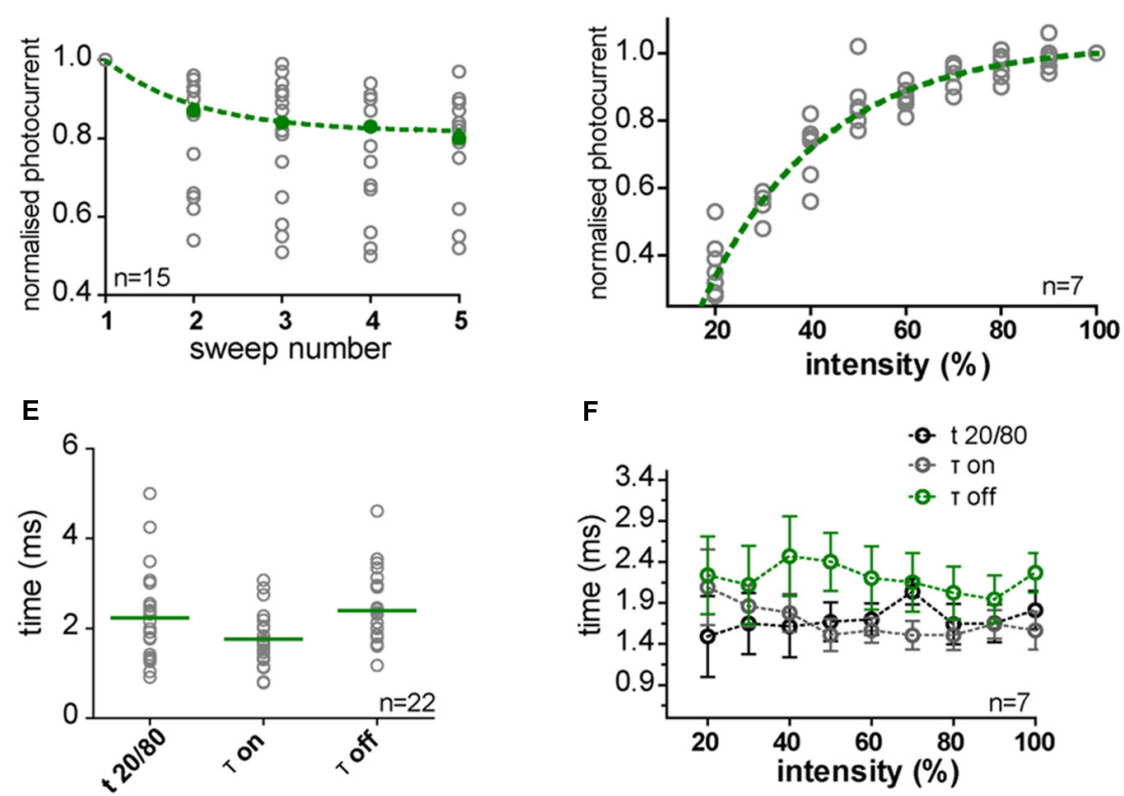

FIGURE 6 | NpHR3.0 photocurrents in transduced IC and MNTB neurons. (A) Photocurrent traces evoked in a transduced IC neuron by 500-ms light pulses (yellow bar) with increasing light power (10\% steps). The photocurrents saturated for light powers above $50 \%$ of the maximum, and slowly declined during the pulse. (B) The decline (over the duration of the pulse) in photocurrents evoked by maximal light power (peak, gray circles; steady-state, light gray circles) in IC ( $n=15)$ and MNTB $(n=8)$ neurons was statistically significant $(p<0.0001$, paired one-tailed $t$-test). (C) Photocurrents normalized to the current of the first light pulse (gray circles) decreased with repeated stimulation, and this decline can be described by an exponential decay (green dashed line). The green filled circles represent the median of the normalized photocurrents. (D) Photocurrents normalized to the response at maximal light power (gray circles) depend on light intensity (Spearman's correlation test, $r=1, p<0.0001$ ) in an exponential fashion (green dashed line). (E) Kinetics of the photocurrents as characterized by $t_{20 / 80}, \tau_{\text {on }}$ and $\tau_{\text {off }}$ measured at maximal light power. Gray circles represent individual measurements and green horizontal strokes depict the mean values. (F) Photocurrent kinetics was independent of light intensity (Spearman's correlation test: $t_{20 / 80}, r=0.6000 ; p=0.0968 ; \tau_{\text {on }}, r=-0.6333 ; p=0.0760 ; \tau_{\text {off }}, r=-0.3333 ; p=0.3853$ ). Error bars indicate the standard deviation.

an important auditory model that has hitherto been inaccessible to optogenetic manipulations. More than $50 \%$ of the neurons within the targeted region were found to have been transduced, which is comparable to values reported for the mouse brain using AAV2/8 (Cearley and Wolfe, 2006; McFarland et al., 2009; Cardin et al., 2010). Adequate expression levels were detected as early as 7 days after injection, in agreement with expression times of one to several weeks reported in the literature (Cardin et al., 2010; Adamantidis et al., 2011; Yizhar et al., 2011b). In our study, expression persisted for several weeks, becoming slightly more efficient over that period. The success of stereotactic injection, as indicated by expression of the transduced vector, varies with the anatomical location of the nucleus. The lower values for DNLL and MNTB in comparison to the $100 \%$ success rate in the IC are most probably attributable to the smaller physical size and deeper positions of the former ( $\sim 5.5 \mathrm{~mm}$ from lambda for DNLL; 8.2-8.4 mm for MNTB). Moreover, inter-individual differences in cranial anatomy become more important with longer injection paths, further reducing stereotactical precision and efficiency. Their small particle size of only $20 \mathrm{~nm}$ facilitates diffusion of AAVs, allowing them to transduce large target areas; even in case of imprecise injections. One advantage of AAV8(Y733F) is its lack of axonal transport [which has been reported for other AAVs in the mouse (Taymans et al., 2007; Zheng et al., 2009; Masamizu et al., 2011; Castle et al., 2014)], which would otherwise have limited targeting specificity. 


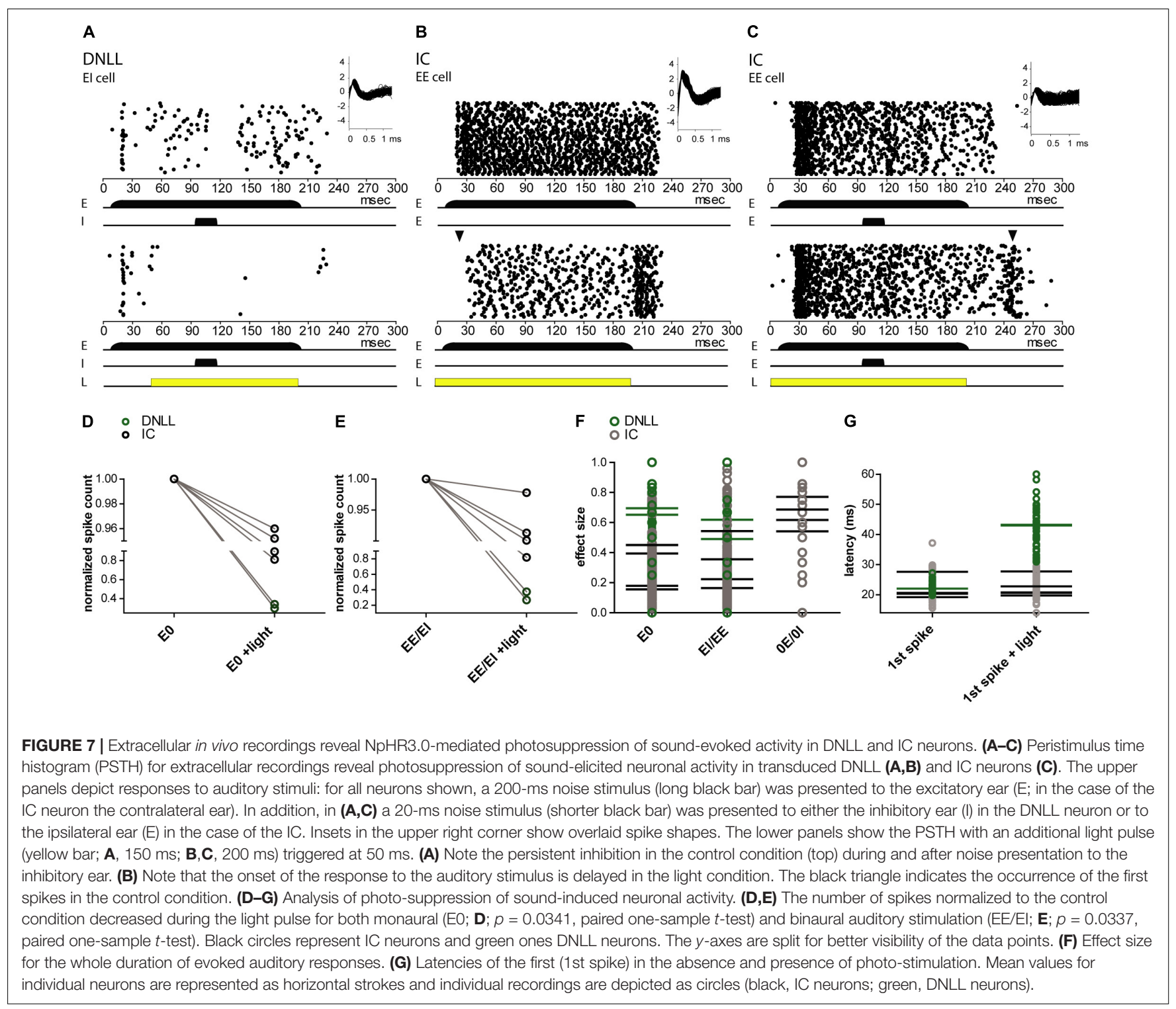

\section{Optical Control of Neuronal Activity in IC, MNTB, and DNLL of the Gerbil}

Our in vitro electrophysiological characterization of transduced brain slices demonstrates that rAAV vectors are a useful tool for functional and neuron-specific expression of $\mathrm{CatCH}$ and NpHR3.0 in the gerbil. By $10 \mathrm{dpi}$, APs could be elicited or suppressed with light. The most important stimulation parameter was pulse duration and to some degree it can be utilized to compensate for variations in expression levels. Longer light pulses decreased the latencies and jitter of elicited APs, making firing not only more efficient but also faster. The latencies determined in our in vitro recordings were similar to published values (Kleinlogel et al., 2011). Latencies of the first and subsequent APs exhibited no differences, which is an important prerequisite for precise high-frequency photostimulation. No increase in phase locking to preferred stimulation frequencies was encountered in IC neurons (Langner and Schreiner, 1988); instead, pulse duration was the determinant parameter for phase locking. The use of very long light pulses resulted in extra spikes or depolarization block, which is characterized by a plateau potential, decreasing AP amplitudes and incomplete repolarization. The mechanisms underlying the depolarization block are not completely understood, but most likely large cation currents leading to prolonged membrane depolarization are involved (Bianchi et al., 2012). The degree of depolarization block varies between neuronal cell types. It is assumed to be lowest in neurons with high spiking frequencies and low input resistances (Herman et al., 2014).

The temporal fidelity of photo-excitation using $\mathrm{CatCH}$ did not match the high frequency and temporal precision seen in auditory neurons, since light pulse widths limit the achievable maximum frequency. Though some neurons could be driven with light at $100 \mathrm{~Hz}$ in the absence of any extra spikes, most neurons reacted only to pulse trains at $40-50 \mathrm{~Hz}$; these frequencies 
are nevertheless higher than those usually reported for $\mathrm{CatCH}$ (Kleinlogel et al., 2011). However, using the vector introduced here for neuron-specific expression in the gerbil brain together with faster channelrhodopsins such as Chronos (Klapoetke et al., 2014), it should be possible to bring stimulation frequencies closer to the physiological maxima of auditory neurons.

The recorded NpHR3.0 photocurrents recorded here, and their kinetics, match published data (Gradinaru et al., 2010). Current amplitudes varied substantially from cell to cell and with light intensities, but kinetics were independent of light intensity. The decrease in photocurrent with repeated stimulation was too small to become detrimental and might be counteracted by flashes of blue light or longer dark periods (Zhang et al., 2007).

\section{Light-Stimulated Suppression of Sensory Evoked in Vivo Firing in Gerbils}

We have successfully established in vivo optogenetics in the gerbil brain. APs evoked by auditory stimuli in DNLL and IC neurons expressing NpHR3.0 were suppressed by light in anesthetized animals. As already stated for in vitro experiments, the duration and timing of light pulses were the most important determinants of efficient optogenetic stimulation. Light pulses triggered shortly before the onset of the sensory stimulus and entirely encompassing it were shown to be the most effective. Light pulses with these properties have also been used in other in vivo studies (Felix-Ortiz et al., 2015). However, light transmission into brain tissue, which is accompanied by losses in light power, remains problematic. To enhance photosuppression, saturating light levels have to be applied, because NpHR3.0 transports only one charge per photon. This was observed in our in vitro recordings at $50 \%$ light intensity $\left(3-4 \mathrm{~mW} / \mathrm{mm}^{2}\right)$, all of which were obtained from cells less than $100 \mu \mathrm{m}$ away from the fiber tip. Usually the fiber was 250-600 $\mu \mathrm{m}$ distant from the recording electrode tip and its output usually amounted to only $0.5-4.5 \mathrm{~mW} / \mathrm{mm}^{2}$, resulting in lower light levels at the recording site. This factor, in combination with cell-tocell variability in NpHR3.0 expression levels, most probably contributes to the variations in suppression efficacy. Another effect that needs to be carefully considered is post-inhibitory rebound spikes observed after cessation of the light pulse both in vitro and in vivo. In patch-clamp experiments these always occurred in neurons that exhibited the hyperpolarizationactivated current $\left(I_{\mathrm{h}}\right)$, which can depolarize the neuron after the end of illumination (Halder et al., 2015). Experimental conditions should account for these side effects and limit the occurrence of rebound spikes to a time frame that lies beyond the actual tested paradigm. This is another reason for choosing a light-pulse duration which more than encompasses that of the tested auditory paradigm. Inter-stimulus intervals must also be sufficiently long to counteract changes in the chloride reversal potential that occur at constant NpHR activity (Raimondo et al., 2012). The chloride reversal potential is maintained by the transporter KCC2, which is present in high levels in the auditory brainstem (Friauf et al., 2011). Therefore, chloride equilibrium is assumed to be quickly reached after NpHR3.0 activation in these nuclei.

\section{CONCLUSION}

We have successfully designed and validated optogenetic tools for use in the brainstem (MNTB and DNLL) and midbrain (IC) of the Mongolian gerbil, one of the leading model organisms for studies of auditory processes. We have demonstrated the importance of detailed in vitro characterization of the optogenetic tools, in terms of kinetic and illumination parameters, in the nuclei in which they will subsequently be used in vivo. As shown here, the viral vector AAV8(Y733F) with the hSyn I promoter enables reliable delivery and expression of genes with neuronal specificity and thus paves the way for studies of unanswered questions in auditory neurobiology in freely behaving animals. Moreover, apart from its use in optogenetics, the viral vector should allow almost any transgene smaller than $4.7 \mathrm{~kb}$ to be expressed in neurons of the Mongolian gerbil and may even permit genome editing with the help of the CRISPR/Cas9 system.

\section{AUTHOR CONTRIBUTIONS}

SK and BB performed the experiments. SK, BB, and LK analyzed the data. SM supervised AAV production. All authors were involved in study design and writing of the manuscript.

\section{FUNDING}

We thank the following organizations for financial support: Graduate School GRK 1091 (German Research Foundation, DFG), International Max Planck Research Schools (IMPRS), Graduate School of Systemic Neurosciences (GSN, LMU Munich), Munich Center for Neurosciences (MCN, LMU Munich), and Collaborative Research Center SFB 870 (German Research Foundation, DFG).

\section{ACKNOWLEDGMENTS}

We would like to thank Fred Koch, Elisabeth Schulze, and Phuong Nguyen for the help with AAV production and Christian Porres for teaching us the art of stereotactic injections. We also thank Mike Myoga, Michael Pecka, and Annette Stange-Marten for their support with in vivo recordings and Julian Ammer for fruitful discussions and advice on data analysis. SK thanks the International Max Planck Research School (IMPRS) for Molecular Life Sciences for support. Data presented in this manuscript were generated by SK and form part of his doctoral thesis at LMU Munich (Keplinger, 2016), which can be accessed online (https://edoc.ub.unimuenchen.de/).

\section{SUPPLEMENTARY MATERIAL}

The Supplementary Material for this article can be found online at: https://www.frontiersin.org/articles/10.3389/fncel.2018. 00111/full\#supplementary-material 


\section{REFERENCES}

Adamantidis, A. R., Tsai, H.-C., Boutrel, B., Zhang, F., Stuber, G. D., Budygin, E. A., et al. (2011). Optogenetic interrogation of dopaminergic modulation of the multiple phases of reward-seeking behavior. J. Neurosci. 31, 10829-10835. doi: 10.1523/JNEUROSCI.2246-11.2011

Ågren, G. (1984). Pair formation in the Mongolian gerbil. Anim. Behav. 32, 528-535. doi: 10.1016/S0003-3472(84)80291-2

Ammer, J. J., Siveke, I., and Felmy, F. (2015). Activity-Dependent transmission and integration control the timescales of auditory processing at an inhibitory synapse. Curr. Biol. 25, 1562-1572. doi: 10.1016/j.cub.2015.04.026

Aschauer, D. F., Kreuz, S., and Rumpel, S. (2013). Analysis of transduction efficiency, tropism and axonal transport of AAV serotypes 1, 2, 5, 6, 8 and 9 in the mouse brain. PLoS One 8:e76310. doi: 10.1371/journal.pone. 0076310

Becirovic, E., Bohm, S., Nguyen, O. N., Riedmayr, L. M., Hammelmann, V., Schon, C., et al. (2016). AAV Vectors for FRET-Based analysis of proteinprotein interactions in photoreceptor outer segments. Front. Neurosci. 10:356. doi: 10.3389/fnins.2016.00356

Bellomo, M., Marini, H., Adamo, E. B., Catania, M. A., Mannucci, C., Squadrito, F., et al. (2006). Vascular endothelial growth factor induces brain erythropoietin expression? Funct. Neurol. 21, 87-91.

Bianchi, D., Marasco, A., Limongiello, A., Marchetti, C., Marie, H., Tirozzi, B., et al. (2012). On the mechanisms underlying the depolarization block in the spiking dynamics of CA1 pyramidal neurons. J. Comput. Neurosci. 33, 207-225. doi: 10.1007/s10827-012-0383-y

Boyden, E. S., Zhang, F., Bamberg, E., Nagel, G., and Deisseroth, K. (2005). Millisecond-timescale, genetically targeted optical control of neural activity. Nat. Neurosci. 8, 1263-1268. doi: 10.1038/nn1525

Broekman, M., Comer, L., Hyman, B., and Sena-Esteves, M. (2006). Adenoassociated virus vectors serotyped with AAV8 capsid are more efficient than AAV-1 or-2 serotypes for widespread gene delivery to the neonatal mouse brain. Neuroscience 138, 501-510. doi: 10.1016/j.neuroscience.2005.11.057

Burger, C., Gorbatyuk, O. S., Velardo, M. J., Peden, C. S., Williams, P., Zolotukhin, S., et al. (2004). Recombinant AAV viral vectors pseudotyped with viral capsids from serotypes 1,2, and 5 display differential efficiency and cell tropism after delivery to different regions of the central nervous system. Mol. Ther. 10, 302-317. doi: 10.1016/j.ymthe.2004.05.024

Cardin, J. A., Carlen, M., Meletis, K., Knoblich, U., Zhang, F., Deisseroth, K., et al. (2010). Targeted optogenetic stimulation and recording of neurons in vivo using cell-type-specific expression of Channelrhodopsin-2. Nat. Protoc. 5, 247-254. doi: 10.1038/nprot.2009.228

Castle, M. J., Gershenson, Z. T., Giles, A. R., Holzbaur, E. L. F., and Wolfe, J. H. (2014). Adeno-associated virus serotypes 1, 8, and 9 share conserved mechanisms for anterograde and retrograde axonal transport. Hum. Gene Ther. 25, 705-720. doi: 10.1089/hum.2013.189

Cearley, C. N., and Wolfe, J. H. (2006). Transduction characteristics of adenoassociated virus vectors expressing cap serotypes 7, 8, 9, and rh10 in the mouse brain. Mol. Ther. 13, 528-537. doi: 10.1016/j.ymthe.2005.11.015

Chen, C. H., McCullagh, E. A., Pun, S. H., Mak, P. U., Vai, M. I., Mak, P. I., et al. (2017). An integrated circuit for simultaneous extracellular electrophysiology recording and optogenetic neural manipulation. IEEE Trans. Biomed. Eng. 64, 557-568. doi: 10.1109/TBME.2016.2609412

Cremer, L. (1948). Geometrische Raumakustik. Hirzel: Stuttgart.

Deisseroth, K., Feng, G., Majewska, A. K., Miesenbock, G., Ting, A., and Schnitzer, M. J. (2006). Next-generation optical technologies for illuminating genetically targeted brain circuits. J. Neurosci. 26, 10380-10386. doi: 10.1523/JNEUROSCI. 3863-06.2006

Felix-Ortiz, A. C., Burgos-Robles, A., Bhagat, N. D., Leppla, C. A., and Tye, K. M. (2015). Bidirectional modulation of anxiety-related and social behaviors by amygdala projections to the medial prefrontal cortex. Neuroscience 321, 197-209. doi: 10.1016/j.neuroscience.2015.07.041

Fenno, L., Yizhar, O., and Deisseroth, K. (2011). The development and application of optogenetics. Annu. Rev. Neurosci. 34, 389-412. doi: 10.1146/annurevneuro-061010-113817

Friauf, E., Rust, M. B., Schulenborg, T., and Hirtz, J. J. (2011). Chloride cotransporters, chloride homeostasis, and synaptic inhibition in the developing auditory system. Hear. Res. 279, 96-110. doi: 10.1016/j.heares.2011.05.012
Goldberg, J. M., and Brown, P. B. (1969). Response of binaural neurons of dog superior olivary complex to dichotic tonal stimuli: some physiological mechanisms of sound localization. J. Neurophysiol. 32, 613-636. doi: 10.1152/ jn.1969.32.4.613

Gradinaru, V., Zhang, F., Ramakrishnan, C., Mattis, J., Prakash, R., Diester, I., et al. (2010). Molecular and cellular approaches for diversifying and extending optogenetics. Cell 141, 154-165. doi: 10.1016/j.cell.2010.02.037

Grothe, B., and Pecka, M. (2014). The natural history of sound localization in mammals - a story of neuronal inhibition. Front. Neural Circuits 8:116. doi: 10.3389/fncir.2014.00116

Haas, H. (1951). Über den Einflu $\beta$ eines Einfachechos auf die Hörsamkeit von Sprache. Acta Acust. United Acust. 1, 49-58.

Halder, S., Van Vliet, K., Smith, J. K., Duong, T. T., McKenna, R., Wilson, J. M., et al. (2015). Structure of neurotropic adeno-associated virus AAVrh.8. J. Struct. Biol. 192, 21-36. doi: 10.1016/j.jsb.2015.08.017

Han, X., and Boyden, E. S. (2007). Multiple-color optical activation, silencing, and desynchronization of neural activity, with single-spike temporal resolution. PLoS One 2:e299. doi: 10.1371/journal.pone.0000299

Herman, A. M., Huang, L., Murphey, D. K., Garcia, I., and Arenkiel, B. R. (2014). Cell type-specific and time-dependent light exposure contribute to silencing in neurons expressing Channelrhodopsin-2. eLife 3:e01481. doi: 10.7554/eLife. 01481

Ito, M., van Adel, B., and Kelly, J. B. (1996). Sound localization after transection of the commissure of Probst in the albino rat. J. Neurophysiol. 76, 3493-3502. doi: 10.1152/jn.1996.76.5.3493

Kandler, K., Clause, A., and Noh, J. (2009). Tonotopic reorganization of developing auditory brainstem circuits. Nat. Neurosci. 12, 711-717. doi: 10.1038/nn.2332

Kelly, J. B., Li, L., and van Adel, B. (1996). Sound localization after kainic acid lesions of the dorsal nucleus of the lateral lemniscus in the albino rat. Behav. Neurosci. 110, 1445-1455. doi: 10.1037/0735-7044.110. 6.1445

Keplinger, S. F. (2016). Establishing Optogenetic Tools in the Auditory System of the Mongolian Gerbil. Doctoral dissertation, LMU München, Munich.

Klapoetke, N. C., Murata, Y., Kim, S. S., Pulver, S. R., Birdsey-Benson, A., Cho, Y. K., et al. (2014). Independent optical excitation of distinct neural populations. Nat. Methods 11, 338-346. doi: 10.1038/nmeth.2836

Kleinlogel, S., Feldbauer, K., Dempski, R. E., Fotis, H., Wood, P. G., Bamann, C., et al. (2011). Ultra light-sensitive and fast neuronal activation with the $\mathrm{Ca}(2)+-$ permeable channelrhodopsin CatCh. Nat. Neurosci. 14, 513-518. doi: 10.1038/ nn.2776

Koch, S., Sothilingam, V., Garcia Garrido, M., Tanimoto, N., Becirovic, E., Koch, F., et al. (2012). Gene therapy restores vision and delays degeneration in the CNGB1(-/-) mouse model of retinitis pigmentosa. Hum. Mol. Genet. 21, 4486-4496. doi: 10.1093/hmg/dds290

Langner, G., and Schreiner, C. E. (1988). Periodicity coding in the inferior colliculus of the cat. I. Neuronal mechanisms. J. Neurophysiol. 60, 1799-1822. doi: 10.1152/jn.1988.60.6.1799

Lingner, A., Wiegrebe, L., and Grothe, B. (2012). Sound localization in noise by gerbils and humans. J. Assoc. Res. Otolaryngol. 13, 237-248. doi: 10.1007/ s10162-011-0301-4

Loskota, W. J., Lomax, P., and Verity, M. A. (1974). A Stereotaxic Atlas of the Mongolian Gerbil Brain. Ann Arbor, MI: Ann Arbor Science Publishers.

Maki, K., and Furukawa, S. (2005). Acoustical cues for sound localization by the Mongolian gerbil, Meriones unguiculatus. J. Acoust. Soc. Am. 118, 872-886. doi: 10.1121/1.1944647

Manley, G. A. (2017). The mammalian cretaceous cochlear revolution. Hear. Res. 352, 23-29. doi: 10.1016/j.heares.2016.12.007

Masamizu, Y., Okada, T., Kawasaki, K., Ishibashi, H., Yuasa, S., Takeda, S., et al. (2011). Local and retrograde gene transfer into primate neuronal pathways via adeno-associated virus serotype 8 and 9. Neuroscience 193, 249-258. doi: 10.1016/j.neuroscience.2011.06.080

McFarland, N. R., Lee, J.-S., Hyman, B. T., and McLean, P. J. (2009). Comparison of transduction efficiency of recombinant AAV serotypes 1, 2, 5, and 8 in the rat nigrostriatal system. J. Neurochem. 109, 838-845. doi: 10.1111/j.1471-4159. 2009.06010.x

Mills, J. H., Schmiedt, R. A., and Kulish, L. F. (1990). Age-related changes in auditory potentials of Mongolian gerbil. Hear. Res. 46, 201-210. doi: 10.1016/ 0378-5955(90)90002-7 
Nagel, G., Brauner, M., Liewald, J. F., Adeishvili, N., Bamberg, E., and Gottschalk, A. (2005). Light activation of channelrhodopsin-2 in excitable cells of Caenorhabditis elegans triggers rapid behavioral responses. Curr. Biol. 15, 2279-2284. doi: 10.1016/j.cub.2005.11.032

Nothwang, H. G. (2016). Evolution of mammalian sound localization circuits: a developmental perspective. Prog. Neurobiol. 141, 1-24. doi: 10.1016/j. pneurobio.2016.02.003

Pecka, M., Brand, A., Behrend, O., and Grothe, B. (2008). Interaural time difference processing in the mammalian medial superior olive: the role of glycinergic inhibition. J. Neurosci. 28, 6914-6925. doi: 10.1523/JNEUROSCI.1660-08.2008

Pecka, M., Zahn, T. P., Saunier-Rebori, B., Siveke, I., Felmy, F., Wiegrebe, L., et al. (2007). Inhibiting the inhibition: a neuronal network for sound localization in reverberant environments. J. Neurosci. 27, 1782-1790. doi: 10.1523/JNEUROSCI.5335-06.2007

Pollak, G. D., Burger, R. M., and Klug, A. (2003). Dissecting the circuitry of the auditory system. Trends Neurosci. 26, 33-39. doi: 10.1016/S0166-2236(02) 00009-7

Raimondo, J. V., Kay, L., Ellender, T. J., and Akerman, C. J. (2012). Optogenetic silencing strategies differ in their effects on inhibitory synaptic transmission. Nat. Neurosci. 15, 1102-1104. doi: 10.1038/nn.3143

Roberts, M. T., Seeman, S. C., and Golding, N. L. (2014). The relative contributions of MNTB and LNTB neurons to inhibition in the medial superior olive assessed through single and paired recordings. Front. Neural Circuits 8:49. doi: 10.3389/ fncir.2014.00049

Ryan, A. (1976). Hearing sensitivity of the Mongolian gerbil, Meriones unguiculatis. J. Acoust. Soc. Am. 59, 1222-1226. doi: 10.1121/1.380961

Salo, A. L., and French, J. A. (1989). Early experience, reproductive success, and development of parental behaviour in Mongolian gerbils. Anim. Behav. 38, 693-702. doi: 10.1016/S0003-3472(89)80015-6

Schuller, G., Radtke-Schuller, S., and Betz, M. (1986). A stereotaxic method for small animals using experimentally determined reference profiles. J. Neurosci. Methods 18, 339-350. doi: 10.1016/0165-0270(86)90022-1

Shimazaki, K., Urabe, M., Monahan, J., Ozawa, K., and Kawai, N. (2000). Adenoassociated virus vector-mediated bcl-2 gene transfer into post-ischemic gerbil brain in vivo: prospects for gene therapy of ischemia-induced neuronal death. Gene Ther. 7, 1244-1249. doi: 10.1038/sj.gt.3301211

Shneiderman, A., Oliver, D. L., and Henkel, C. K. (1988). Connections of the dorsal nucleus of the lateral lemniscus: an inhibitory parallel pathway in the ascending auditory system? J. Comp. Neurol. 276, 188-208. doi: 10.1002/cne.902760204

Siveke, I., Pecka, M., Seidl, A. H., Baudoux, S., and Grothe, B. (2006). Binaural response properties of low-frequency neurons in the gerbil dorsal nucleus of the lateral lemniscus. J. Neurophysiol. 96, 1425-1440. doi: 10.1152/jn.00713.2005

Spitzer, M. W., and Semple, M. N. (1991). Interaural phase coding in auditory midbrain: influence of dynamic stimulus features. Science 254, 721-724. doi: $10.1126 /$ science. 1948053

Spitzer, M. W., and Semple, M. N. (1995). Neurons sensitive to interaural phase disparity in gerbil superior olive: diverse monaural and temporal response properties. J. Neurophysiol. 73, 1668-1690. doi: 10.1152/jn.1995.73.4.1668

Taymans, J.-M., Vandenberghe, L. H., Haute, C. V. D., Thiry, I., Deroose, C. M., Mortelmans, L., et al. (2007). Comparative analysis of adeno-associated viral vector serotypes 1, 2, 5, 7, and 8 in mouse brain. Hum. Gene Ther. 18, 195-206. doi: 10.1089/hum.2006.178

Tollin, D. J. (2003). The lateral superior olive: a functional role in sound source localization. Neuroscientist 9, 127-143. doi: 10.1177/1073858403252228

Wallach, H., Newman, E. B., and Rosenzweig, M. R. (1949). The precedence effect in sound localization. Am. J. Psychol. 62, 315-336. doi: 10.2307/1418275

Willaredt, M. A., Schluter, T., and Nothwang, H. G. (2015). The gene regulatory networks underlying formation of the auditory hindbrain. Cell. Mol. Life Sci. 72, 519-535. doi: 10.1007/s00018-014-1759-0

Yizhar, O., Fenno, L. E., Davidson, T. J., Mogri, M., and Deisseroth, K. (2011a). Optogenetics in neural systems. Neuron 71, 9-34. doi: 10.1016/j.neuron.2011. 06.004

Yizhar, O., Fenno, L. E., Prigge, M., Schneider, F., Davidson, T. J., O’Shea, D. J., et al. (2011b). Neocortical excitation/inhibition balance in information processing and social dysfunction. Nature 477, 171-178. doi: 10.1038/nature10360

Zhang, F., Gradinaru, V., Adamantidis, A. R., Durand, R., Airan, R. D., de Lecea, L., et al. (2010). Optogenetic interrogation of neural circuits: technology for probing mammalian brain structures. Nat. Protoc. 5, 439-456. doi: 10.1038/ nprot.2009.226

Zhang, F., Wang, L. P., Brauner, M., Liewald, J. F., Kay, K., Watzke, N., et al. (2007). Multimodal fast optical interrogation of neural circuitry. Nature 446, 633-639. doi: 10.1038/nature05744

Zheng, H., Qiao, C., Wang, C.-H., Li, J., Li, J., Yuan, Z., et al. (2009). Efficient retrograde transport of adeno-associated virus type 8 to spinal cord and dorsal root ganglion after vector delivery in muscle. Hum. Gene Ther. 21, 87-97. doi: 10.1089/hum.2009.131

Zhong, L., Li, B., Mah, C. S., Govindasamy, L., Agbandje-McKenna, M., Cooper, M., et al. (2008). Next generation of adeno-associated virus 2 vectors: point mutations in tyrosines lead to high-efficiency transduction at lower doses. Proc. Natl. Acad. Sci. U.S.A. 105, 7827-7832. doi: 10.1073/pnas.080286 6105

Zincarelli, C., Soltys, S., Rengo, G., and Rabinowitz, J. E. (2008). Analysis of AAV serotypes 1-9 mediated gene expression and tropism in mice after systemic injection. Mol. Ther. 16, 1073-1080. doi: 10.1038/mt.2008.76

Zorio, D. A. R., Monsma, S., Sanes, D. H., Golding, N. L., Rubel, E. W., and Wang, Y. (2018). De novo sequencing and initial annotation of the Mongolian gerbil (Meriones unguiculatus) genome. Genomics. doi: 10.1016/j.ygeno.2018.03.001 [Epub ahead of print].

Conflict of Interest Statement: The authors declare that the research was conducted in the absence of any commercial or financial relationships that could be construed as a potential conflict of interest.

Copyright (c) 2018 Keplinger, Beiderbeck, Michalakis, Biel, Grothe and Kunz. This is an open-access article distributed under the terms of the Creative Commons Attribution License (CC BY). The use, distribution or reproduction in other forums is permitted, provided the original author(s) and the copyright owner are credited and that the original publication in this journal is cited, in accordance with accepted academic practice. No use, distribution or reproduction is permitted which does not comply with these terms. 